\title{
Le système karstique de Sprimont (Belgique). Holotype d'une spéléogenèse par fantômisation
}

\author{
Nicolas Dupont ${ }^{1}$, Yves Quinif ${ }^{1, *}$, Caroline Dubois $^{1}$, Hai Cheng $^{2,3}$ et Olivier Kaufmann ${ }^{1}$ \\ ${ }^{1}$ Service de Géologie Fondamentale et appliquée, Faculté Polytechnique, Université de Mons, rue de Houdain, 9, Mons, Belgique \\ ${ }^{2}$ Minnesota University, Minnesota, USA \\ ${ }^{3}$ Institute of Global Environmental Change. Xi'an Jiaotong University. No. 1 West Building, Xi'an Jiaotong University Qujiang Campus, \\ No. 99 Yanxiang Road, Xi'an, 710054 Shaanxi, China
}

Accepté le 7 décembre 2017

\begin{abstract}
Résumé - Le système karstique de Sprimont est riche de phénomènes karstiques variés dans un contexte géologique bien défini : un synclinal de carbonates carbonifères entourés au nord, à l'est et au sud par des formations famenniennes imperméables. À l'ouest, l'Ourthe recoupe le synclinal perpendiculairement à son axe d'allongement dans le cadre d'un relief appalachien typique et constitue le niveau de base karstique. Le relief consiste en un vallon orienté est-ouest dont l'axe coïncide avec celui du synclinal. Les ruisseaux qui descendent des hauteurs imperméables vers le vallon se perdent après leur entrée dans le domaine calcaire. Ces ruisseaux confluent sous terre pour résurger au « Trou Bleu », unique résurgence du système, non loin du talweg de l'Ourthe. Le système compte de nombreuses grottes, de dimensions souvent décamétriques. Quelques-unes sont de plus grande ampleur, dont la grotte du « Noû Bleû » récemment découverte, qui est un regard sur la rivière souterraine à quelques dizaines de mètres en amont de la résurgence. Les carrières ont dévoilé en plus de diverses grottes des phénomènes karstiques de type fantôme de roche. La karstogenèse de type fantôme de roche consiste en une altération modérée des carbonates produisant une séparation de phase. D'une part, la phase soluble comprend essentiellement le calcium, le magnésium, le bicarbonate et la silice colloïdale. Elle sort du système par la voie souterraine. D'autre part, une altérite résiduelle constitue la phase solide restante comprenant une partie des carbonates cinétiquement moins solubles comme la calcite sparitique et la dolomie pro parte, les insolubles comme les minéraux argileux, le quartz, et la matière organique. Cette phase solide évolue de façon isovolume lors d'une première étape et voit donc sa porosité augmenter, de même que sa fragilité mécanique. Cette première étape se déroule durant une période géologique où le potentiel hydrodynamique est très réduit, la fantômisation s'exerçant grâce à des circulations phréatiques très lentes mais chimiquement agressives. On obtient ainsi des volumes soit totalement circonscrits dans la masse rocheuse (pseudoendokarsts) soit sous forme de couloirs descendant du toit de la roche mère, remplis par l'altérite résiduelle. À Sprimont, la carrière du Coreux a dévoilé ces fantômes de roche. Durant une seconde phase, lorsqu'apparaît un potentiel hydrodynamique suite à une surrection et à l'incision des rivières, l'altérite peut être mécaniquement érodée par des circulations fluviatiles : les grottes «spéléologiques » se forment. Dans le site étudié, une cavité, la grotte «Nico», a été ouverte par l'avancée du front de la carrière. On y trouve une coupe sédimentaire montrant l'altérite résiduelle ravinée par des formations fluviatiles. Cet article décrit la coupe levée dans ces formations au travers de la lithostratigraphie, de la granulométrie et de la minéralogie des grains. La grotte « Nico » résulte de la coalescence de deux grottes superposées, le plancher rocheux séparant les deux cavités étant fantômisé et affaissé. Les parois et le sol sont des fantômes de roche dont l'altérite résiduelle est essentiellement formée de grains dolomitiques, tels les encrines dolomitisées. Les formations fluviatiles ravinent l'altérite suivant une surface chenalisante. Les grains sont constitués de grains remaniés d'altérite, calcite et dolomite, et d'une faible partie d'insolubles tels des grains de muscovite et de quartz en provenance des psammites famenniens. La série détritique est coiffée par un plancher stalagmitiques dont deux échantillons ont été datés de $53851 \pm 2493$ et $61542 \pm 1235$ ans B.P. ce qui situe l'érosion de l'altérite à une date relativement récente dans le Pléistocène supérieur. Ces phénomènes éclairent d'un jour nouveau la formation des grottes
\end{abstract}

\footnotetext{
*Auteur de correspondance : yves.quinif2@gmail.com;

yves.quinif@umons.ac.be
} 
en Haute Belgique. L'évolution du karst de Sprimont trouve son origine dans un massif fantômisé partiellement érodé au cours de la surrection plio-quaternaire. L'apparition du potentiel hydrodynamique responsable de cette érosion trouve son origine dans les différences d'altitude entre pertes supérieures et résurgence à l'aval par enfoncement du niveau de base : la rivière Ourthe. Nous sommes confrontés à un holotype de spéléogenèse dans le cadre d'un relief appalachien. La grotte "Nico » et les phénomènes associés permettent de concevoir un modèle d'évolution des karsts de la Haute Belgique. Enfin, cet exemple montre aussi qu'une distinction claire peut être faite entre le terme karstogenèse qui englobe la fantômisation de celui de spéléogenèse qui ne concerne que le creusement des grottes spéléologiques par érosion mécanique de l'altérite résiduelle.

Mots clés : karstogenèse / fantômes de roche / spéléogenèse / système karstique perte-résurgence / sédiments souterrains

Abstract - Karstic system of Sprimont (Belgium). Holotype of a speleogenesis by ghost-rock karstification. Karstic system of Sprimont shows varied karstic phenomena in a very well defined geological context: a syncline of Carboniferous carbonates enclosed at the north, east and south by impermeable Famennian formations. The axis of the syncline has evolved like a small valley. At the west, Ourthe River cut perpendicularly this syncline in a typical Appalachian relief and constitutes the karstic basis level. Little rivers flow from the Famennian formations to the valley of Sprimont which follows the axis of the syncline. They disappear in swallow holes named "chantoirs" in the local language as soon as they arrive in contact with the carbonates. Tracing tests prove that these rivers merge underground to resurge at the "Trou Bleu", which constitutes the resurgence of the system, near the Ourthe River. Many caves have been discovered, often of modest dimensions except the recent discovery of the cave "Noû Bleû". On the other hand, quarries have opened karstic ghost-rock phenomena. Ghost-rock karstogenesis consists in an in situ weathering of the limestone with a phase separation. The soluble part, essentially calcium, magnesium, bicarbonate and colloidal silica, goes out of the system. The residual alterite is constituted by sparitic calcite, dolomite pro parte and insoluble part like quartz, clay and organic matter. This material, the "ghost-rock", is very porous and mechanically fragile. In a first stage, this evolution takes place with the same initial volume. This first stage of karstification happens during a geological period where there is no hydrodynamical potential. The underground waters flow in the phreatic zone very slowly. They don't erode the residual alterite and only the soluble elements go out of the system. This ghost-rock can be studied in the walls of the Coreux quarry. During a second phase, if a new hydrodynamics potential appears due to a surrection with incision of the rivers, the underground waters can erode mechanically the residual alterite by subterranean rivers circulation. A "speleological" cave is created with a "normal" karstic evolution. In Sprimont, the Coreux quarry have also opened cavities outcome from initial ghost-rock whose a part of the residual alterite has been eroded to form a cave. In particular, the "Nico" cave was cut by the quarry in which a geological section shows the residual alterite capped above a discordance by river deposits and speleothems. Thus, this erosion is due to underground river circulation. The target of this presentation is to establish the lithostratigraphy of this geological section and make the granulometry with morphology and mineralogy of the grains. The lithostratigraphy analysis shows that the "Nico" cave is the result of the coalescence between two superposed caves. The wall between these two caves has been weathered in ghost-rock. A great part of the residual alterite has been eroded by an underground river which has brought river sediments. The granulometry and mineralogy prove this origin. We find removed grains of alterite like crinoids. The grains are constituted by calcite, dolomite and a little part of insoluble minerals like clays. The sedimentary series are capped by a flowstone which has been dated: $53.851 \pm 2.493$ and $61.542 \pm 1.235 \mathrm{yr}$ B.P. This situates the erosion of the alterite and the hollowing of the cave from a recent period. Those phenomena bring very important informations about the genesis and evolution of the karst in Upper Belgium. The recent evolution of the karst of Sprimont is characterized by two types of phenomena from the ghostrocks. First, partial regressive erosion erodes mechanically the residual alterite from the basic level. At downstream, we have a great cave near the resurgence: the cave of "Noû Bleû". In this case, the process of alterite erosion is the best succeeded. Second, at upstream of the system, the compaction of the alterite in the vadose zone generates some voids which can favor the birth of swallow holes. Those lost of rivers participate also to the erosion of the alterite. Our present observations in the "Nico" cave result from this phenomenon. After the first phase of karstification with the formation of a ghost-rock, the apparition of a potential, due to the surrection of the Ardenne massif with the incision of rivers, permits to the underground waters to erode mechanically the residual alterite. Thus, we have here a holotype of the speleogenesis in the frame of an Appalachian relief. The cave "Nico" and the associated phenomena permit to extract a model of evolution of the karsts of Upper Belgium. This example shows too that we can make the difference between the term of karstogenesis which consists in the ghost-rock karstification and the speleogenesis which is the opening of caves by erosion of the residual alterite.

Keyword: karstogenesis / ghost-rock / karstic system / speleogenesis / swallow-holes-resurgence / underground sediments 


\section{Introduction}

Le système karstique de Sprimont présente des phénomènes karstiques variés dans un contexte géologique strictement délimité : un synclinal de carbonates dinantiens entourés au nord, à l'est et au sud de formations imperméables famenniennes. À l'ouest, l'Ourthe recoupe perpendiculairement ce synclinal en relief typiquement appalachien et constitue le niveau de base karstique. Outre des grottes pénétrables, dont le Noû Bleû découvert en 2013 (Ek et al., 2014a, b), des carrières ont trépané des phénomènes karstiques de type fantômes de roche (Quinif, 2010; Quinif et al., 2014; Dubois et al., 2014a). Les phénomènes karstiques de ce vallon apportent une information capitale sur la genèse et l'évolution des phénomènes karstiques de la Haute Belgique. En effet, la carrière du Coreux dévoile des cavités d'une part issues d'un fantôme de roche initial et dont la vidange de l'altérite est due aux travaux d'exploitation, d'autre part des grottes dans lesquelles l'altérite résiduelle est encore présente, mais partiellement érodée par des dépôts endokarstiques fluviatiles. L'évolution du karst de Sprimont est ainsi caractérisée par une érosion partielle régressive de l'altérite résiduelle des fantômes de roche à partir du niveau de base d'une part et tassement de l'altérite à partir de l'amont d'autre part. Nous sommes donc en présence d'un holotype de la spéléogenèse dans le cadre d'un relief appalachien. L'objectif de cet article est de décrire les coupes découvertes à la carrière du Coreux, les phénomènes associés et d'en inférer une évolution des karsts de la Haute Belgique. On peut ainsi faire une distinction entre la karstogenèse qui comprend l'ensemble des phénomènes depuis la fantômisation initiale de la spéléogenèse qui consiste en la formation de grottes spéléologiquement pénétrables formées par érosion de l'altérite résiduelle du fantôme de roche.

\section{Le paysage}

\subsection{Le relief}

Le synclinal de Chanxhe-Sprimont est situé à l'extrémité est du Condroz, à environ $20 \mathrm{~km}$ au Sud de la ville de Liège (Belgique) (Fig. 1). Ce paysage est caractéristique du Condroz: l'axe du système est un vallon subséquent qui suit parallèlement dans une direction W-E le plan axial du synclinal de Sprimont (Fig. 2b). Suivant le relief condruzien habituel, la dépression est incisée dans les carbonates dinantiens entre deux collines (les «tiges » condruziens) sculptées dans les grès et psammites famenniens.

Le vallon de Sprimont est tributaire de l'Ourthe, rivière épigénétique principale de la région qui recoupe perpendiculairement l'axe du synclinal. Ces rivières constituent les axes principaux du réseau hydrographique de la Haute Belgique, résultant d'un enfoncement épigénétique par surimposition d'un réseau hydrographique conséquent suite à la surrection de l'Ardenne (Ek, 1957; Demoulin, 1995). Les ruisseaux descendent des hauteurs famenniennes vers le talweg. La nature karstique du synclinal est exprimée par la perte de l'ensemble de ces ruisseaux pénétrant dans le vallon (Peeters et al., 2012). La sortie du système karstique est unique: la résurgence du Trou Bleu, située à proximité de l'Ourthe (Van den Broeck et al., 1910; Briffoz, 1983; De Broyer et al.,
1996; Meus et al., 2014). Actuellement, la dynamique du système karstique est illustrée par la rapidité des écoulements au sein de l'aquifère calcaire (Nys, 1929; Briffoz, 1983) et par le recul progressif des points de pertes vers l'amont (Ek, 1976; Peeters et al., 2012).

\subsection{Géologie du site}

\subsubsection{Lithostratigraphie}

Le synclinal de Chanxhe-Sprimont est composé de formations essentiellement calcaires datant du Carbonifère inférieur (Fig. 2a; Fourmarier, 1954; Bellière et Marion, 2009; Poty et al., 2002; Mottequin et Marion, 2012, 2014):

- après la formation carbonatée de Comblain-au-Pont appartenant au Dévonien terminal, un premier ensemble tournaisien est constitué des calcaires crinoïdiques de la Formation d'Hastière (HAS), des calcschistes de la Formation du Pont d'Arcole (PDA) et des calcaires crinoïdiques de la Formation de Landelies (LAN);

- le Tournaisien se poursuit avec les calcaires gris-bleu de la Formation d'Yvoir (YVO), à grains moyens à fins et contenant des cherts noirs, répartis irrégulièrement dans les couches. Dans la zone d'étude, le sommet de la formation est généralement très dolomitique;

- l'encrinite de la Formation de l'Ourthe (OUR), de teinte gris-bleu à bleu-noir, est exposée en bancs métriques à plurimétriques et exploitée sous le nom de «Petit-Granit». Contrairement aux formations qui l'encadrent, celle-ci ne contient aucun chert;

- les calcaires à cherts de la Formation de Martinrive (MRT) sont bleu-foncés à noirs, à grain fin et disposés en bancs pluridécimétriques;

- les dolomies de la Formation de Longpré (LPR) à teinte brunâtre proviennent d'une encrinite dolomitisée;

- le Viséen commence avec la brèche calcaire des Formations de Terwagne et de Neffe dite aussi Brèche de la Belle-Roche (BBR).

Par l'intermédiaire d'un passage calcschisteux de 10 à $15 \mathrm{~m}$ d'épaisseur, les calcaires carbonifères reposent sur les grès et siltites du Famennien qui affleurent sur l'ensemble du pourtour du synclinal. La fin de cette série détritique est marquée par un passage progressif à la série carbonatée qui la surplombe. Mis à part les alluvions de la vallée de l'Ourthe et les anciennes terrasses fluviatiles de l'Ourthe avec galets roulés, les formations de couverture consistent en des dépôts sableux cénozoïques d'origine marine conservés dans des cryptokarsts.

\subsubsection{Tectonique}

Le synclinal de Chanxhe-Sprimont a son axe orienté sensiblement Est-Ouest $\left(\mathrm{N} 80^{\circ} \mathrm{E}\right)$; il possède un ennoiement de l'Est vers l'Ouest, principalement marqué au niveau de sa terminaison périsynclinale entre Damré et Sprimont (Fig. 2b). Le synclinal est asymétrique : les pendages sur le flanc sud sont quasi verticaux (pied Nord), tandis que sur le flanc Nord les pendages sont pied Sud et varient de $50^{\circ}$ à $70^{\circ}$. Le synclinal est situé dans la partie orientale du Synclinorium de Dinant. Cette 


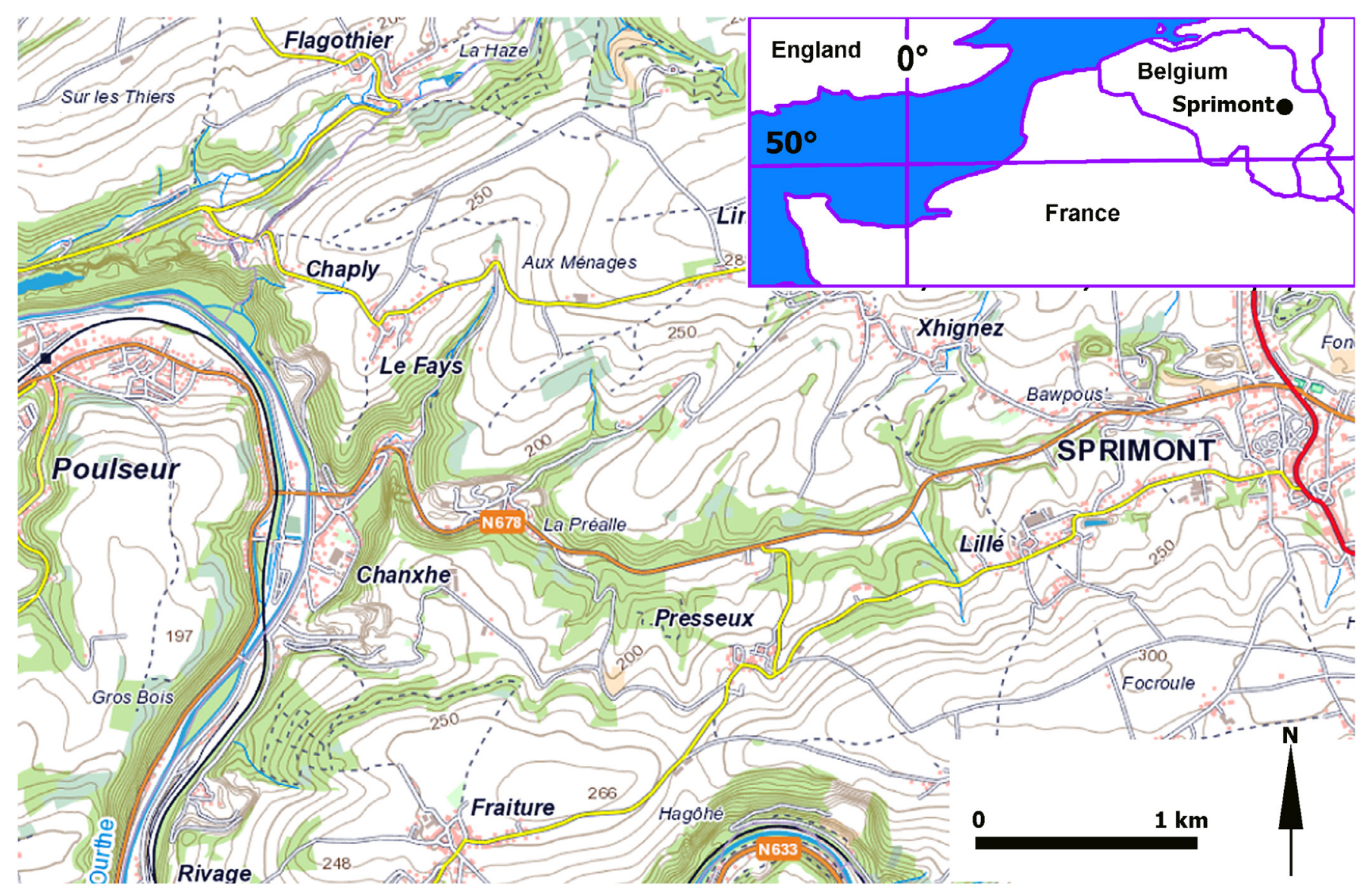

Fig. 1. Topographie de la zone étudiée. Extrait de l'Atlas Topographique Belgique, IGN Ed.

Fig. 1. Topography of the studied area. From "Atlas Topographique Belgique", IGN Ed.

structure plicative, formée lors de l'orogénèse varisque, conditionne le relief caractéristique du Condroz avec les vallons calqués sur les synclinaux calcaires et les crêtes sur les anticlinaux composés de grès et de psammites famenniens.

Deux familles de failles sont présentes dans la région (Ruthy, 2004) : les failles longitudinales, inverses, d'orientation Est-Ouest, parallèles à la stratification, formées lors de l'orogénèse varisque et les failles transverses, orientées NordSud à Nord-Est - Sud-Ouest plus ou moins perpendiculaires à la direction des plis et présentant des rejets faibles, qui seraient liées à une tectonique plus récente.

\subsection{Circulations karstiques}

Le synclinal de Chanxhe-Sprimont comprend une série de petits ruisseaux, s'écoulant depuis les crêtes famenniennes et s'engouffrant rapidement essentiellement dans la Formation d'Hastière au travers de pertes dénommées localement «chantoirs». Certains se perdent un peu plus tôt dans la Formation de Comblain-au-Pont, la dernière du Famennien. D'autres arrivent jusqu'au creux du vallon. Néanmoins, ce dernier ne connaît plus de circulation pérenne continue sur toute la longueur du talweg. L'ensemble des eaux ressortent en aval en un point unique, dénommé Trou Bleu, à proximité de l'Ourthe à Chanxhe.
Jusqu'à la récente découverte de la grotte du Noû Bleû, les grottes et gouffres reconnus n'avaient pas donné accès à un drain (Dubois, 1982; SPW-DGARN, 2005). Ce sont soit des réseaux supérieurs comme le complexe «Enfer-Fissures », soit des avens comme la grotte Espérance, soit de petites cavités recoupées par les carrières. La grotte du Noû Bleû, par contre, est une importante cavité située en aval du système karstique. Elle se compose de vides abandonnés par des écoulements permanents (galeries et plusieurs grandes salles) et d'autres parcourus par une rivière souterraine (Ek et al., 2014a, b). D'après Vergari (2014), cette grotte serait un exemple-type de réactivation partielle d'un fantôme de roche.

\section{Le site de la Carrière du Coreux}

\subsection{Aspect général des phénomènes observés}

La carrière du Coreux est située dans la moitié amont du système. Lors de la découverte des phénomènes observés (2011 et 2012), elle était constituée de deux fosses emboîtées principales qui excavent profondément les calcaires tournaisiens sous la surface du sol (Fig. 3). Elle a trépané de nombreux phénomènes karstiques dont celui qui constitue l'objet principal de cet article. 


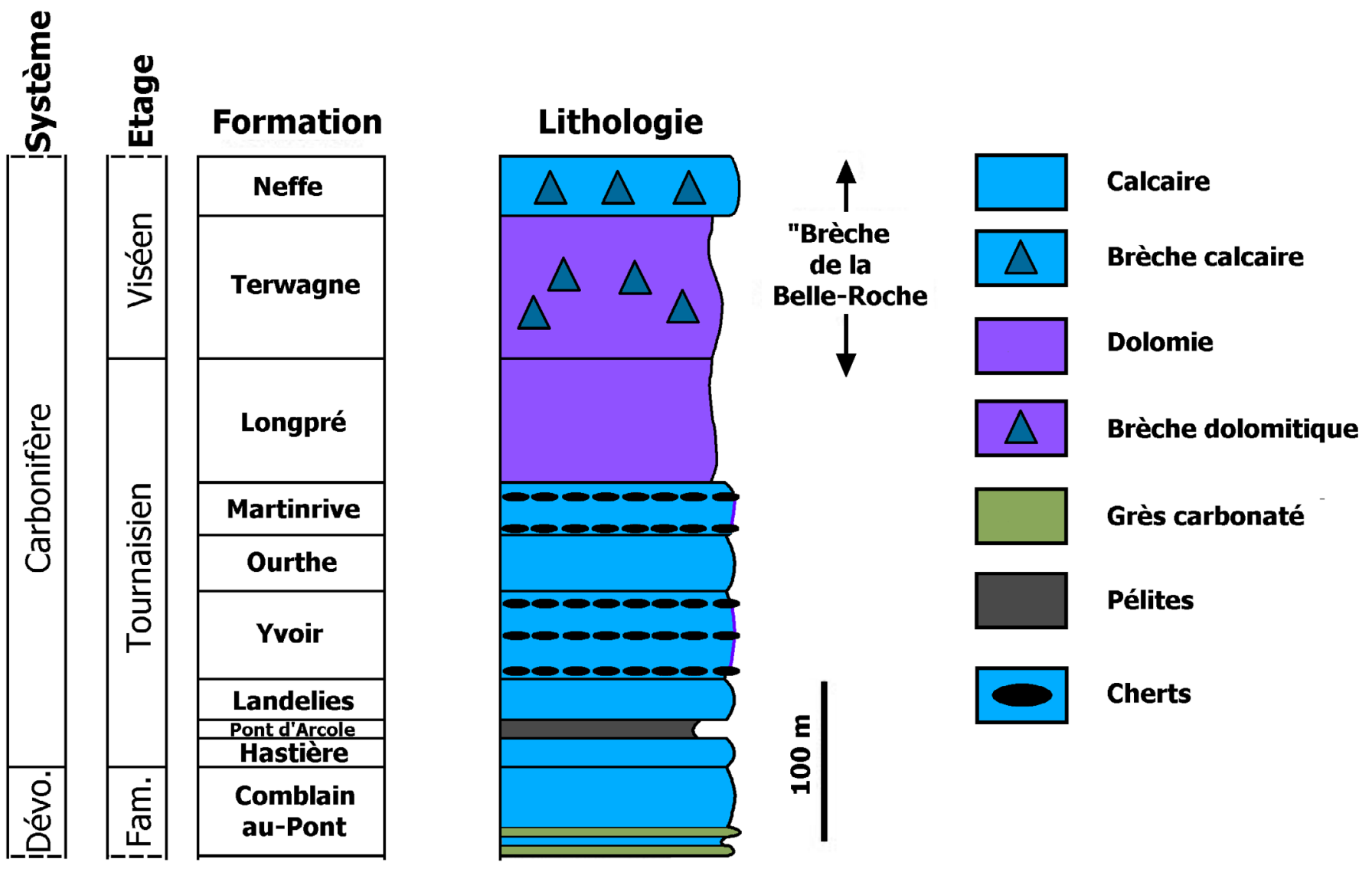

Fig. 2a. Lithostratigraphie de la zone étudiée. Les phénomènes observés se trouvent dans les formations d'Yvoir (grotte « Nico ») et de l'Ourthe (d'après Mottequin et Marion, 2012).

Fig. 2a. Lithostratigraphy of the studied area. The observed phenomena situate in the Formations of Yvoir (Cave "Nico") and Ourthe (after Mottequin et Marion, 2012).

Plusieurs grottes peu étendues ont pu être explorées et topographiées par le CRSOA (Briffoz, 1983). Les fronts ont dégagé plusieurs sites où une altération de la roche mère prouve l'existence de fantômes de roche (Dupont et al., 2012, 2016). Dans la Formation de l'Ourthe, à l'extrémité ouest de la carrière du Coreux, des fractures montrent des épontes altérées, les entroques étant mises en relief par dissolution de la partie micritique (Fig. 4a). Ce type de phénomène avait déjà été constaté par Van den Broeck, Martel, et Rahir (1910), ces auteurs ayant déduit qu'il restait sur place un résidu de dissolution partielle. C'est un stade précoce de la fantômisation: depuis les lèvres, le calcaire massif est altéré sur 1 à $2 \mathrm{~cm}$ de profondeur. Il s'agit du stade II d'altération, «légèrement altéré », selon la terminologie de Price (2009). Certaines fractures sont altérées sur quelques dizaines de centimètres. Par endroits, la fantômisation est telle que la roche devient pulvérulente suite à l'action de phénomènes météoriques. En particulier, un gradient d'altération plus poussé a été observé le long d'une diaclase subverticale d'orientation N-S (Fig. 5). Les fossiles ont particulièrement bien enregistré cette phase avec les clastes encore cohérents dans une matrice pulvérulente (Fig. 4b). Selon la classification proposée par Price (2009), les stades d'altération observables vont du stade II au stade IV. Des mesures de porosité réalisées le long d'un profil transversal au fantôme de roche comme présenté par Dubois et al. (2014b) donnent les résultats donnés dans le tableau 1, en tenant compte que la porosité de la roche saine est de l'ordre de $5 \%$ au maximum. Les analyses minéralogiques en diffraction de rayons $\mathrm{X}$ des roches prélevées le long du même profil montrent que la roche du stade II est composée essentiellement de calcite et d'une faible teneur en dolomite (quelques pourcents). Au stade III, la teneur en dolomite a sensiblement diminué, ce qui suggère l'altération préférentielle de ce minéral vis-à-vis de la calcite. Les analyses d'argile ne montrent la présence que d'illite pour tous les stades d'altération.

Une série de tirs sur le front nord a dégagé des phénomènes de plus grande ampleur dans la Formation d'Yvoir (Fig. 6). Il s'agit de cavités complexes comprenant à la fois des parties altérées et des cavités partiellement remplies de sédiments endokarstiques détritiques et stalagmitiques (Fig. 7). C'est dans une des plus importantes, la grotte «Nico», qu'a été levée la « coupe du coquelicot» décrite au paragraphe suivant. Cette grotte fait partie d'une série d'autres cavités qui s'échelonnent horizontalement suivant les mêmes bancs situés dans la partie sommitale de la Formation d'Yvoir. La présence de ces cavités a été soulignée lors d'un tir durant lequel une importante partie du front s'est affaissée brutalement dans la carrière. 


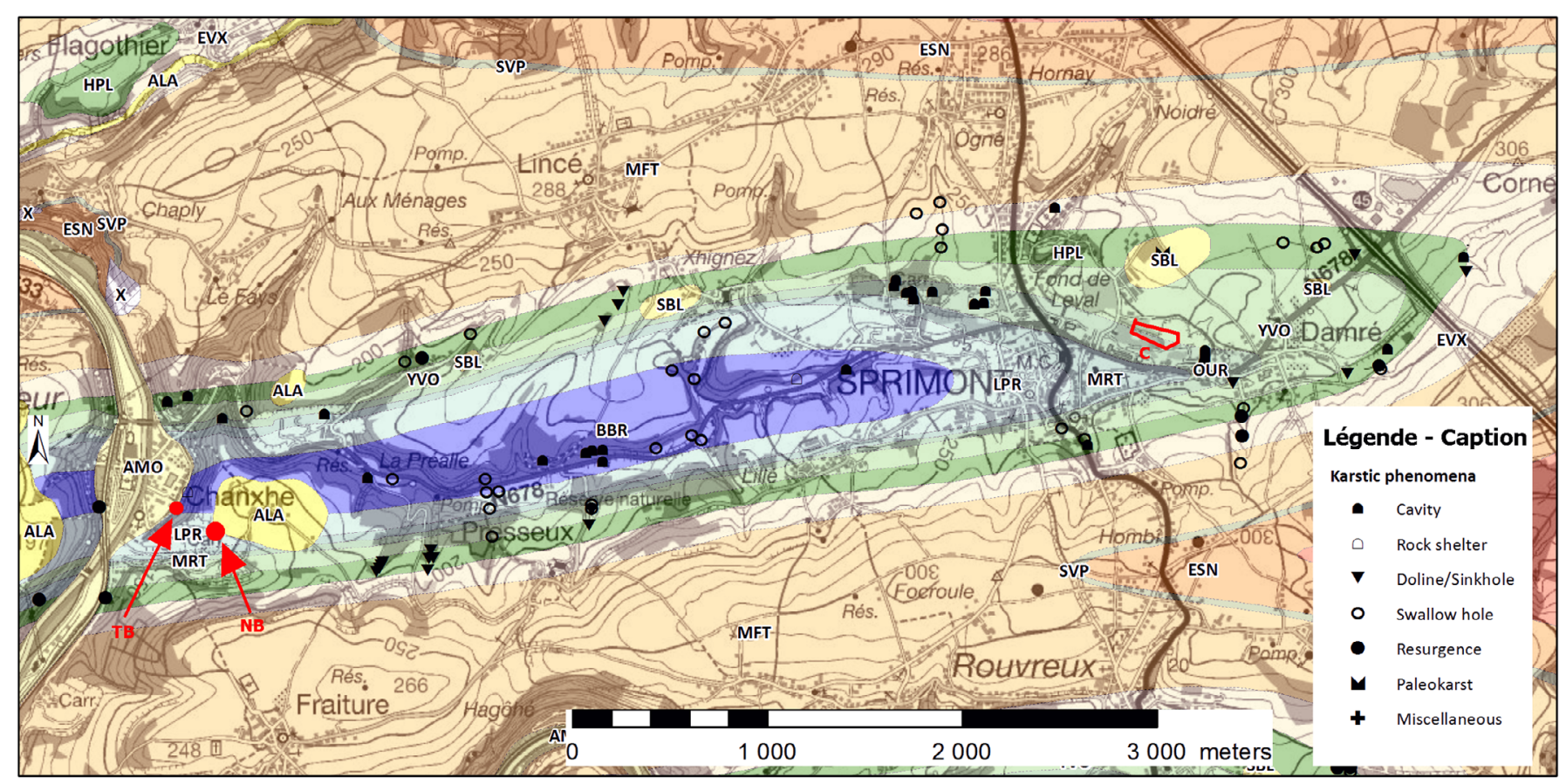

Fig. 2b. Carte géologique de la zone étudiée (d'après Mottequin et Marion, 2012, 2014). Famennien avec : ESN : Formation d'Esneux; SVP : Formation de Souverain-Pré; MFT: Formation de Montfort; EVX : Formation d'Évieux et de Comblain-au-Pont. Tournaisien avec: HPL : Formations d'Hastière, Pont d'Arcole et Landelies ; YVO : Formation d'Yvoir; OUR : Formation de l'Ourthe ; MRT : Formation de Martinrive ; LPR : Formation de Longpré. Viséen avec : BBR : Formation de la Belle Roche regroupant les Formations de Neffe et de Terwagne. SBL : sables landéniens; ALA : alluvions anciennes; AMO: Alluvions modernes. X: terrains anthropiques. C: carrière du Coreux. TB: Trou bleu, résurgence générale du système. NB: grotte du Noû Bleû.

Fig. 2b. Geological map of the studied area (following Mottequin et Marion, 2012, 2014). Famennian with: ESN: Formation of Esneux; SVP: Formation of Souverain-Pré; MFT: Formation of Montfort; EVX: Formation of Evieux and of Comblain-au-Pont. Tournaisian with: HPL: Formations of Hastière, Pont d'Arcole and Landelies; YVO: Formation of Yvoir; OUR: Formation of Ourthe; MRT: Formation of Martinrive; LPR: Formation of Longpré. Visean with: BBR: Formation of la Belle Roche regrouping the Formations of Neffe and of Terwagne. SBL: Thanetian sands; ALA: old alluvium; AMO: modern alluvium. X: human terranes. C: Coreux quarry. TB: general resurgence of the system ("Trou Bleu"). NB: Cave of "Noû Bleû".

\subsection{Lithostratigraphie de la coupe du coquelicot}

\subsubsection{Situation de la coupe}

La coupe du coquelicot se situe au front de la grotte «Nico », une courte cavité ouverte par minage. Au-dessus d'un front haut de 3 à 5 mètres, une petite salle précède quelques amorces de galeries vite obstruées. Les parois montrent des traces de l'altération chimique présente sous un fin concrétionnement qui lui est postérieur, tandis que le sol de cette salle est recouvert de spéléothèmes.

\subsubsection{Description lithostratigraphique}

La coupe s.s. a été levée en deux profils stratigraphiques distants d'un mètre (Fig. 8 et 14). D'une manière générale, le front de cette cavité est constitué d'un massif fantômisé à la base (C), l'altérite étant pulvérulente, surmonté de dépôts limoneux jaune à ocre en plusieurs formations imbriquées (D). Parfois, des blocs éboulés s'intercalent entre ces formations (B). L'ensemble est surmonté d'une formation stalagmitique complexe comprenant un plancher inférieur scellant les formations limoneuses et un plancher supérieur recouvrant une roche fantômisée $(\mathrm{F})$. Cette disposition stratigraphique résulte d'une coalescence entre une cavité supérieure et une cavité inférieure totalement colmatée.
La coupe de gauche est marquée par une formation jaune stratifiée (D) discordante sur un massif fantômisé (C). Ce dernier est pulvérulent, se transformant en poudre au toucher. Il est totalement en place; des filonnets de calcite y sont apparents. Il ne réagit pas à l' $\mathrm{HCl}$. Un pilier d'altérite résiduelle partage la formation jaune en deux parties. Ce fantôme de roche est de même texture que le massif inférieur. Au dessus de la formation jaune, une rangée de blocs altérés compris dans une altérite plus poussée $(B)$ la sépare d'un toit formé de la roche mère perturbée mais toujours stratifiée, assez fantômisée (A).

D'un point de vue macroscopique, la nature du fantôme de roche est confirmée par ces observations. Les filonnets de calcite restant plus cohérents que le reste de la formation résultent d'une altération différentielle entre une partie micritique présentant un fort rapport surface-volume et une partie sparitique dont les plus gros cristaux, avec un rapport surface-volume plus faible, présentent une cinétique de dissolution plus lente. Cette texture a été observée à maintes reprises dans les fantômes de roche fossiles des carrières hennuyères (Quinif et al., 1993; Havron et al., 2007). L'analyse microscopique des grains donnée ci-dessous confirme cette interprétation.

La coupe de droite est plus complexe. On y retrouve toujours l'ensemble stratifié jaune (D) discordant sur la roche 


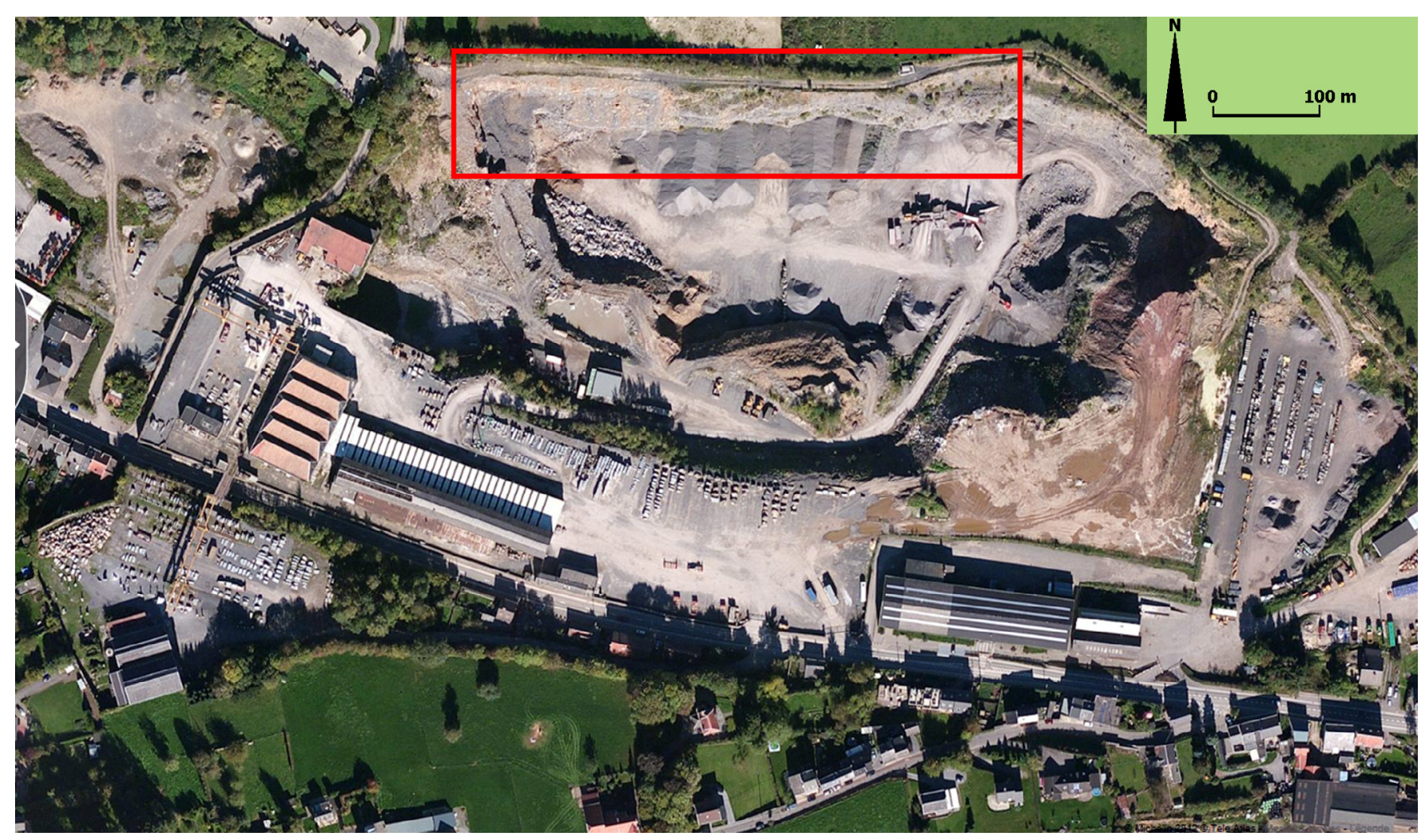

Fig. 3. Vue aérienne de la Carrière du Coreux (cliché Google Earth ${ }^{\oplus}$ ). Les phénomènes karstiques étudiés se localisent sur le flanc nord, souligné par un encadré rouge.

Fig. 3. Aerial view of the Coreux quarry $\left(\right.$ Google Earth $\left.{ }^{\odot}\right)$. Studied karstic phenomena are on the north wall, in the red rectangle.

mère fantômisée $(\mathrm{C})$ dont la texture est légèrement différente de celle de la coupe de gauche. Au dessus de la formation jaune, une strate fantômisée et démantelée (B) supporte une formation argileuse jaune foncé à stratification inclinée $(\mathrm{E})$. Vient ensuite un groupe de blocs fantômisés affaissés supportant une série détritique débutant par une lentille à texture d'argile à blocaux. La série détritique se termine par un dépôt essentiellement constitué d'entroques libres. La série est enfin coiffée par une formation stalagmitique complexe avec une partie inférieure à faciès en «mille feuilles » et une partie supérieure plus massive. On peut interpréter cette série stratigraphique comme résultant de la réunion de deux cavités superposées. La partie inférieure est semblable à la coupe de gauche avec l'altérite résiduelle surmontée en discordance par la formation argilo-limoneuse jaune. La strate démantelée et fantômisée sépare cette cavité inférieure d'une cavité supérieure à l'intérieur de laquelle se sont sédimentés le limon ocre (4) et les formations supérieures ( 5 et 6 ) coiffées par une formation stalagmitique (7 et 8 ).

\subsubsection{Analyse des principales formations}

Les échantillons meubles ont été analysés par granulométrie. Ils ont été lavés sur une série de tamis 80-63-50-40-32-25$20 \mu \mathrm{m}$. Le refus à $80 \mu \mathrm{m}$ a été tamisé à sec sur la série de tamis 3150-2500-2000-1600-1250-1000-800-630-500-400-315-250200-160-125-100 $\mu \mathrm{m}$. Les refus ont été pesés et récupérés pour une analyse sous binoculaire. Enfin, une analyse diffractométrique aux rayons $\mathrm{X}(\mathrm{DRX})$ sur poudre et dépôt orienté a été effectuée pour quelques échantillons. Une remarque préliminaire s'impose. La granulométrie de l'altérite résiduelle a été réalisée dans le but de retrouver les fractions granulométriques susceptibles d'avoir été emportées et resédimentées par un courant d'eau. D'éventuelles conclusions hydrodynamiques à partir de la granulométrie de l'altérite résiduelle n'ont aucun sens puisque cette analyse porte sur des formations autochtones, sans aucun transport.

\subsubsection{Le fantôme de roche}

La fantômisation atteint plusieurs niveaux d'altération, depuis les roches encore cohérentes mais présentant une plus forte porosité jusqu'à l'altérite pulvérulente mais toujours en place. Les échantillons 1 et 9 appartiennent à cette dernière catégorie (Fig. 8). De fins filons témoignent de l'altération in situ de la roche mère (Fig. 9).

Les courbes granulométriques (Fig. 10) montrent un mode principal autour de $140-100 \mu \mathrm{m}$ qui exprime la nature des grains ayant mieux résisté à l'altération : les entroques. D'une façon générale, les paramètres granulométriques expriment la nature pétrographique et la texture de la roche mère biodétritique. Les moyennes des deux échantillons sont de 235 et $151 \mu \mathrm{m}$. Deux types de paramètres expriment la parenté des deux échantillons: le refus à $50 \mu \mathrm{m}$ (90 et $84 \%$ ) et les paramètres de forme : rapport entre l'écart-type et la moyenne (222 et $213 \%$ ), Qdphi $(0,47$ et 0,53$)$, skewness $(5,2$ et 5,7$)$ et curtosis (31 et 38 ). 


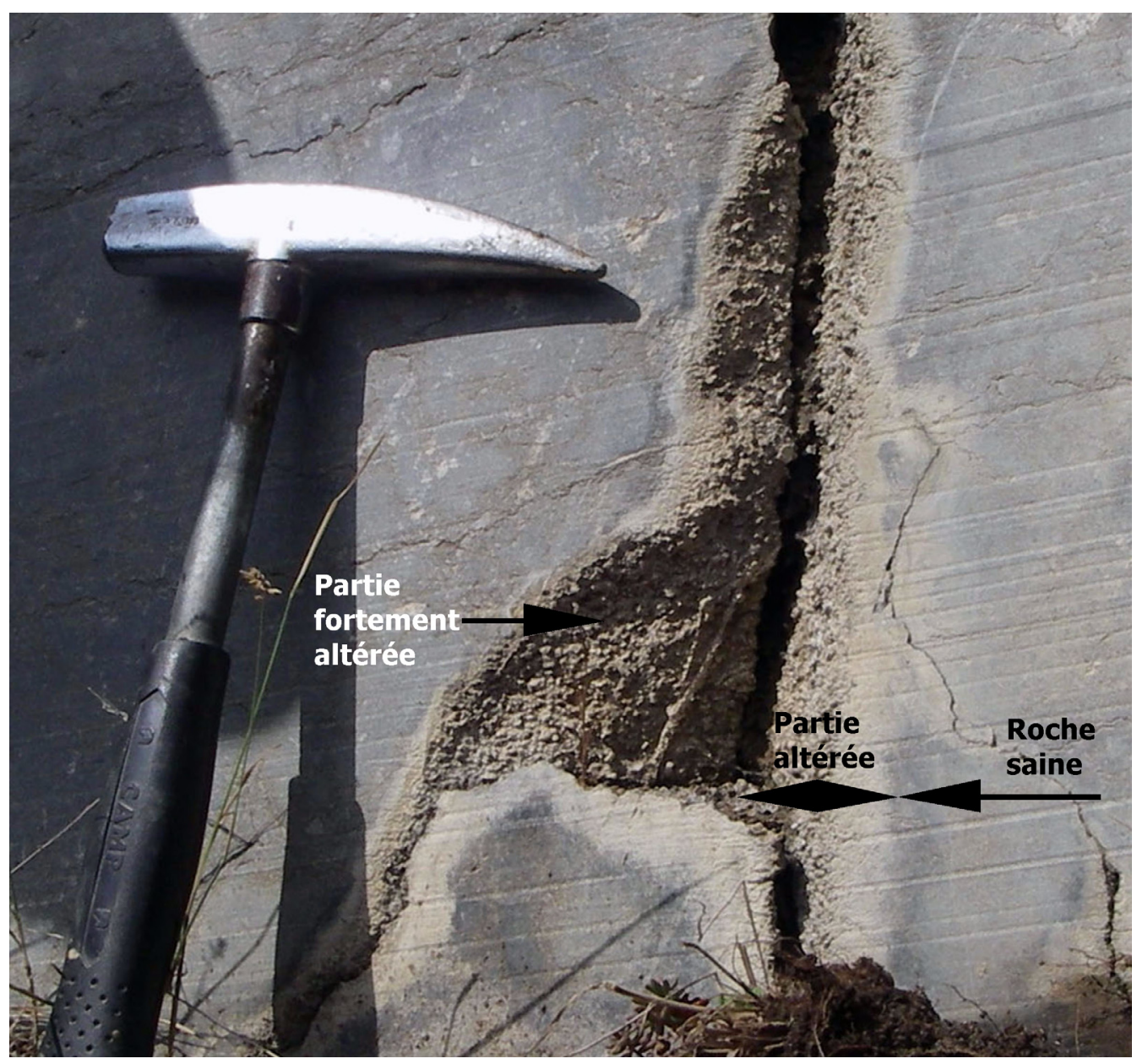

Fig. 4a. (à gauche). Fracture fantômisée. L'aspect granuleux des surfaces altérées provient de la mise en relief des entroques, constituant essentiel de ce «Petit-Granit». Les plages plus claires de la roche expriment le gradient d'altération.

Fig. 4a. Ghost-rock on fracture. The grain aspect of the weathered surfaces is due to the expression of the crinoids constituting essentially the limestones "Petit-Granit". The weathered gradient is expressed by the lighter surfaces of the rock.

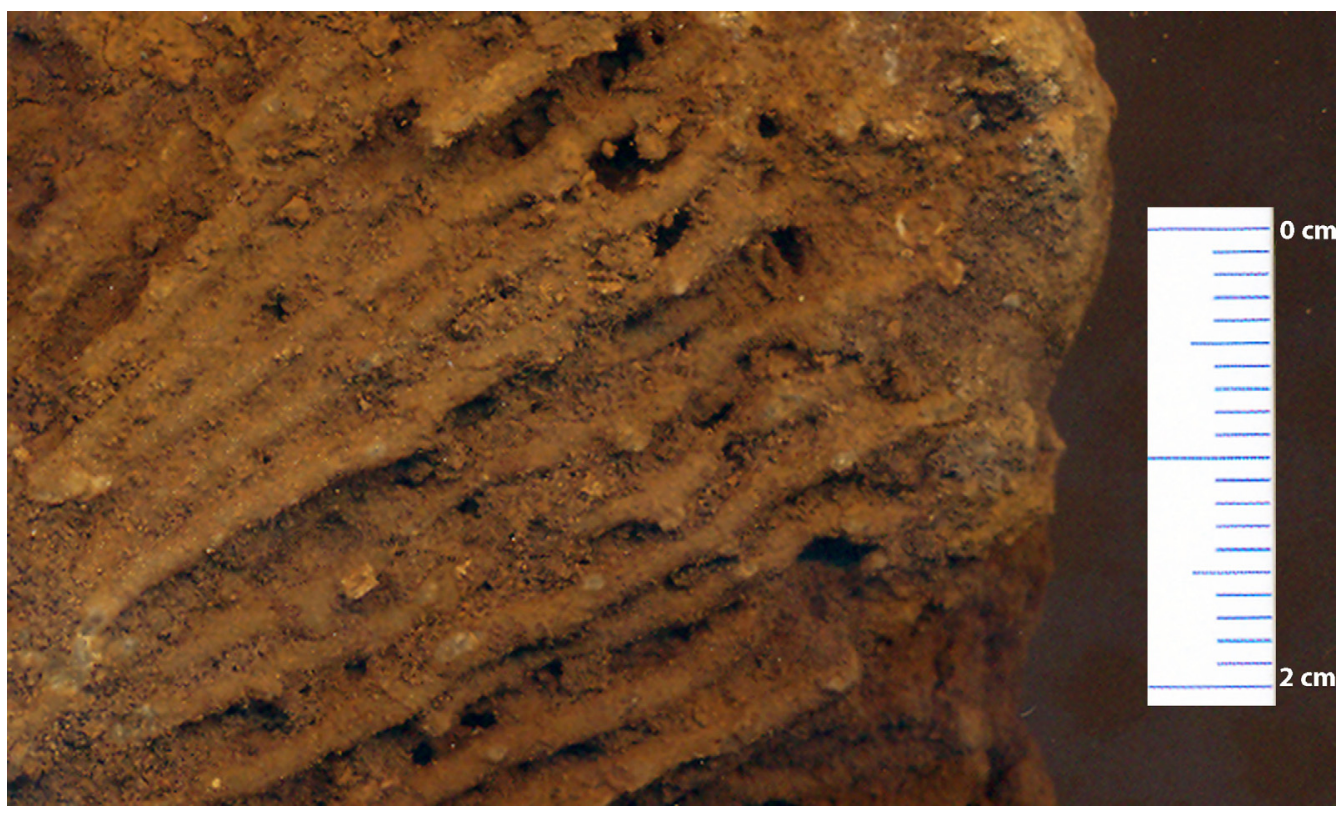

Fig. 4b. Fossile de Syringopora fantômisé. Les clastes du fossile sont encore cohérents mais la matrice est pulvérulente. Fig. 4b. Fossil of a weathered Syringopora. The clasts of the fossil are yet well-knit but the matrix is crumbled. 
L'examen à la loupe binoculaire des fractions granulométriques confirme cette constatation en apportant d'autres informations. Certains des fins filons refusés au-dessus de la fraction à $2500 \mu \mathrm{m}$ réagissent à $\mathrm{l}^{\prime} \mathrm{HCl}$, d'autres pas. Les entroques sont présents sous forme de tubes au-dessus de $2500 \mu \mathrm{m}$; ils sont constitués de petits rhomboèdres qui hérissent la surface de ces tubes. Beaucoup de ces cristaux rhomboédriques ne réagissent pas à l'acide, d'autres oui. Les fractions inférieures à $1000 \mu \mathrm{m}$ sont surtout formées de grains cristallins provenant des encrines dissociées. Lorsqu'on observe une attaque ménagée par $\mathrm{HCl}$ sous binoculaire, on voit qu'une partie des grains se dissolvent, certains restent

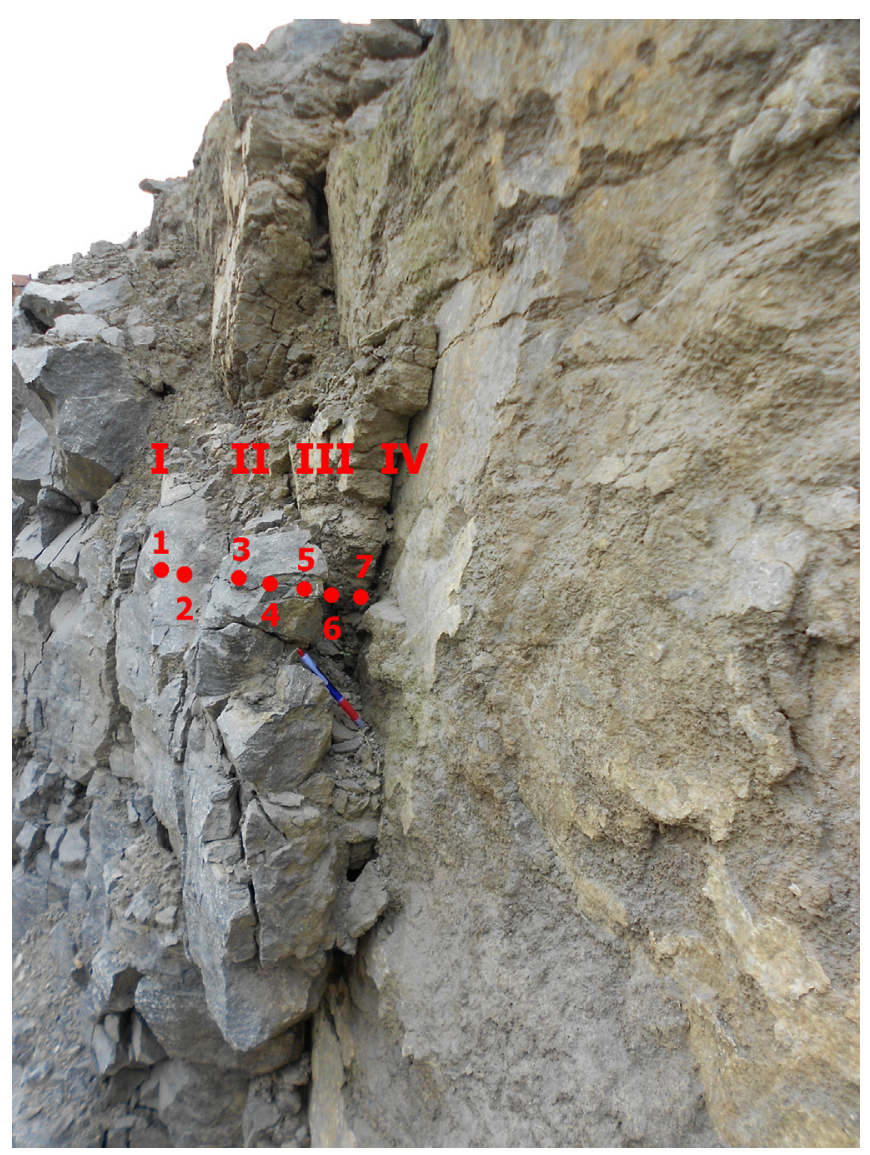

Fig. 5. Répartition des zones d'altération.

Fig. 5. Distribution of the weathered areas. totalement insolubles. Sur les grains restant insolubles, des tâches brunes peuvent apparaître. Une observation analogue est faite sur les fractions très fines. À $50 \mu \mathrm{m}$, L'attaque par $\mathrm{HCl}$ d'un petit tas de cristaux, transparents, opaques et translucides, donne une réaction assez importante au début avec une «mousse de bulles». Des grains bruns s'isolent. La réaction s'atténue puis des îlots de grains apparaissent qui restent sans réaction apparente malgré un ajout d'HCl.

L'analyse en DRX sur poudre confirme cette analyse (Tab. 2) et permet d'identifier la dolomite comme principal minéral. Notons la présence de quartz peu visible sur les fractions granulométriques.

L'altérite est donc formée d'entroques et débris d'entroques surtout constitués de dolomite, avec néanmoins des cristaux résiduels de calcite. Cette composition est conforme avec la nature dolomitique de la roche mère non altérée échantillonnée près de la coupe étudiée.

Ces fantômes de roche constituent la matière première à la circulation fluviatile tardive qui a pu éroder et mobiliser l'altérite. Il faut donc s'attendre à retrouver certains de ces éléments dans les formations fluviatiles.

\subsubsection{La formation détritique jaune}

Cette formation est ravinante sur l'altérite basale. Deux échantillons ont été analysés, à la base (2) et au sommet (3). Ce sont des limons argileux dont les paramètres sont indiqués au tableau 3 et à la figure 11 .

L'analyse de Passega (Fig. 12) place ces échantillons un peu au dessous de la zone de suspension uniforme (Passega, 1957, 1964, 1977 ; Passega et Byramjee, 1969). Ces sédiments résultent donc de la sédimentation de particules non classées près du fond par un courant légèrement turbulent.

Les grains des fractions grossières peu abondantes présentent un faciès semblable à ceux des fantômes de roche, avec des débris d'entroques. De plus, une quinzaine de grains de quartz, translucides et mats, peu émoussés à anguleux ont été dénombrés. Les autres grains sont en dolomite. Les fractions fines sont constituées de grains de quartz et de muscovite, ce qu'indique clairement l'analyse en DRX sur poudres (Tab. 2). Les grains de quartz sont parfois émoussés mais restent assez anguleux. La muscovite est plus abondante dans des fractions au dessus de $32 \mu \mathrm{m}$ qu'en dessous.

L'analyse en DRX sur dépôts orientés fournit les résultats suivants: échantillon 2: $60 \%$ Illite- $40 \%$ interstratifiés irréguliers, échantillon 3: $75 \%$ Illite $-25 \%$ interstratifiés irréguliers. Ces minéraux sont caractéristiques d'un sédiment

Tableau 1. Mesures de porosité le long d'un profil perpendiculaire au fantôme de roche.

Table 1. Measurements of porosity along a profile perpendicular to the ghost-rock.

\begin{tabular}{|c|c|c|c|c|}
\hline Stades d'altération (Price, 2009) & Échantillon & Masse volumique $\left(\mathrm{gr} / \mathrm{cm}^{3}\right)$ & Porosité $(\%)$ & Teneur massique en $\mathrm{CaCO} 3(\%)$ \\
\hline \multirow[t]{2}{*}{ I. Non altéré } & 1 & 2,56 & 5,4 & 97 \\
\hline & 2 & 2,51 & 9,2 & 97 \\
\hline II. Légèrement altéré & 4 & 2,4 & 13,7 & 96 \\
\hline III. Moyennement altéré & 5 & 2,26 & 18 & 95 \\
\hline
\end{tabular}




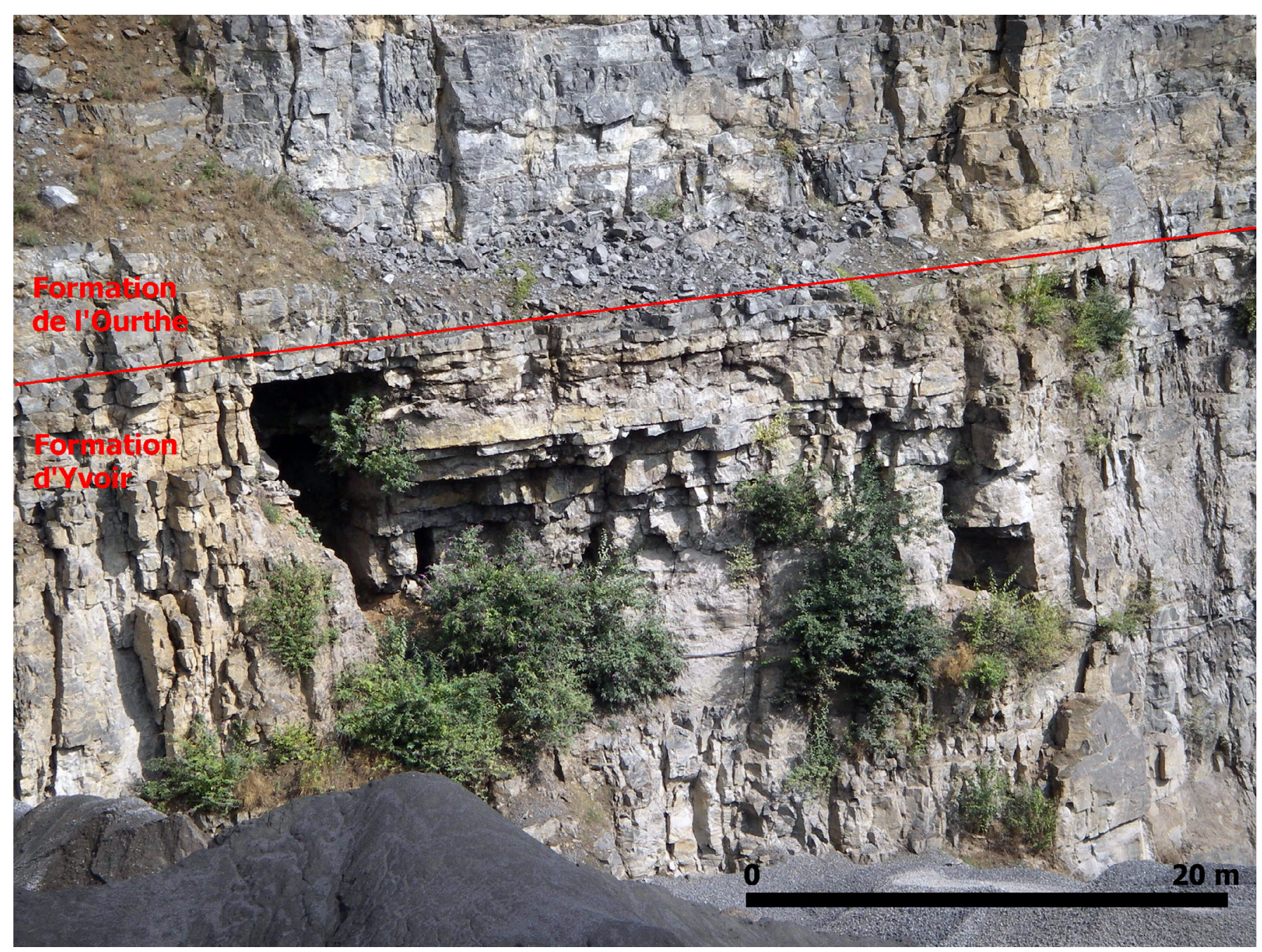

Fig. 6. Cavités dans le front nord de la Carrière du Coreux. Elles se situent dans la partie sommitale de la Formation d'Yvoir sous la Formation de l'Ourthe.

Fig. 6. Cavities in the north wall of the Coreux quarry. They are situated in the upper part of the Formation of Yvoir, under the Formation of Ourthe.

chimiquement peu évolué ce qui confirme l'origine de l'érosion des roches famenniennes.

Cette formation est donc constituée à la fois de sédiments provenant de l'érosion mécanique de fantômes de roche mais surtout de grains provenant des psammites famenniens. Le vecteur est un courant d'eau : une rivière souterraine apportant les résultats de l'érosion des psammites en amont du système karstique et les débris de l'altérite karstique, prouvant ainsi une formation de grotte spéléologique par enlèvement d'une partie de l'altérite résiduelle des fantômes de roche.

\subsubsection{Les formations détritiques supérieures de la coupe de droite (Fig. 13)}

La formation ocre (échantillon 4) a une courbe très dispersée, difficile d'interprétation. Par contre, les formations supérieures (échantillons 5 et 6) dessinent des courbes analogues à celle des altérites (Fig. 13 et 14). Dans ce cas, il s'agit clairement de ces altérites remaniées, plus particulièrement les altérites de l'échantillon 9 à deux modes voisins.
Il faut néanmoins noter que le limon ocre possède également de façon très atténuée les deux modes de l'altérite, en plus d'autres modes. Les paramètres granulométriques se trouvent dans le tableau 4.

Le diagramme de Passega de ces formations (Fig. 12) distingue clairement le limon ocre (échantillon 4) qui ne correspond à aucune plage classique. La présence de gros grains (premier centile élevé), de l'absence de classement clair et d'une médiane faible indique un sédiment mis en place sans remaniement important. L'échantillon 5 se situe entre les dépôts de suspension uniforme (RS) et de suspension gradée $(\mathrm{QR})$. La déconvolution fait apparaître un mode plus proche des dépôts de décantation totale (T). Cette formation indique l'existence d'un courant trop faible pour produire un classement, avec des épisodes provoquant une décantation. L'échantillon 6 est caractéristique d'un courant plus fort, entraînant des grains de plus de $1 \mathrm{~mm}$. La déconvolution (6a et $6 \mathrm{~b}$ ) situe un mode dans les dépôts de suspension gradée, l'autre sous les dépôts de suspension uniforme (Fig. 12) . 


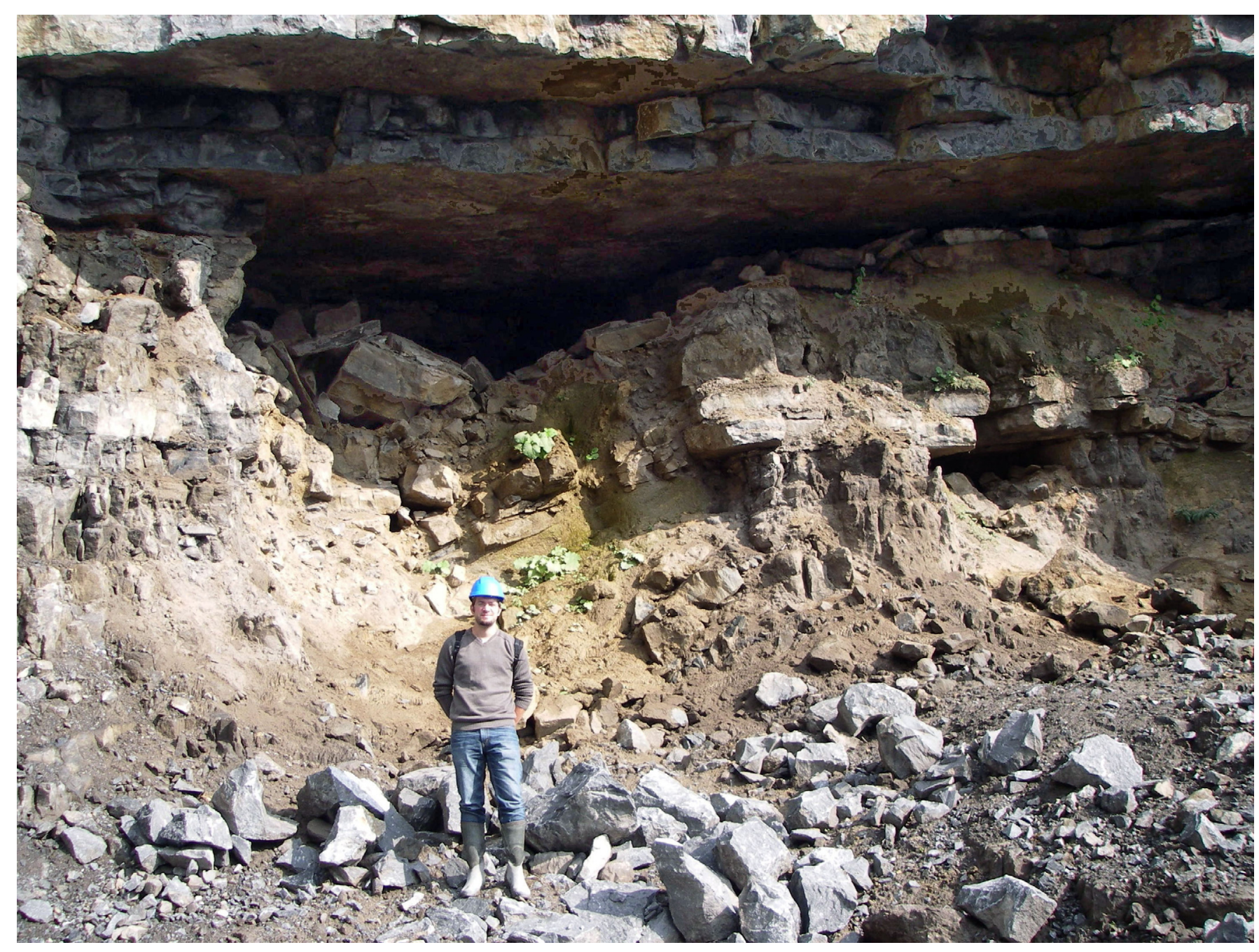

Fig. 7. La grotte « Nico». On distingue de haut en bas la cavité ouverte avec concrétions stalagmitiques, l'ensemble sédimentaire étudié ci-après et des éboulis surtout dus aux travaux de la carrière.

Fig. 7. The cave "Nico". From the top to the bottom: the opened cavity with speleothems, the studied sedimentary formation and fallen blocks which are due to the quarry works.

L'examen à la loupe binoculaire de la fraction granulométrique à $500 \mu \mathrm{m}$ de la formation ocre (échantillon 4) montre un faciès analogue à celui du fantôme de roche avec des entroques et débris d'entroques. Certains sont altérés. On ne voit plus que les cristaux de dolomite qui les hérissent, avec une forte porosité. Quelques cristaux blancs transparents roulés ou émoussés sont composés de calcite : ils sont totalement dissous dans $1^{\prime} \mathrm{HCl}$. La réaction à $\mathrm{HCl}$ sur un échantillonnage indifférencié est assez vive. La solution devient brunâtre. Les entroques hérissés restants ont une très faible réactivité. La fraction à $100 \mu \mathrm{m}$ est surtout composée de grains blancs opaques à jaunâtres. Ils ont tous l'apparence de rhomboèdres avec des microfacettes cristallines. On ne trouve pas de grains émoussés ou arrondis d'apparence de quartz. La réaction à l'HCl est quasiment inexistante. Il s'agit donc de grains de dolomite. À $25 \mu \mathrm{m}$ par contre, des grains lamellaires trahissent la présence de débris de muscovite. En l'absence de réaction à $l^{\prime} \mathrm{HCl}$, cette fraction est formée de dolomite, quartz et muscovite. Quant à l'échantillon 6, toutes les fractions sont essentiellement constituées de dolomite, soit sous forme d'entroques et de débris d'entroques pour les plus grossières, soit de cristaux de dolomite et de grains de quartz pour les fractions fines. La plupart de ces grains présentent de traces d'érosion ou de corrosion.

\subsubsection{La formation stalagmitique terminale}

Son existence fondamentale prouve une évolution morphosédimentaire endokarstique «classique» en présence d'un corps géologique fantômisé (Fig. 15). L'absence de ce plancher dans la coupe de gauche montre que la coupe de droite est en fait une coalescence entre au moins deux cavités. Elles proviennent de l'érosion mécanique partielle de l'altérite résiduelle du fantôme de roche. Deux échantillons stratigraphiquement successifs ont été datés par la méthode du déséquilibre radioactif dans la famille de l'uranium-238 (Tab. 5).

Les âges trouvés sont surprenants par rapport au contexte climatique car ils se situent dans le stade isotopique 4 , le début de la dernière glaciation. Ils sont également jeunes, témoignant 

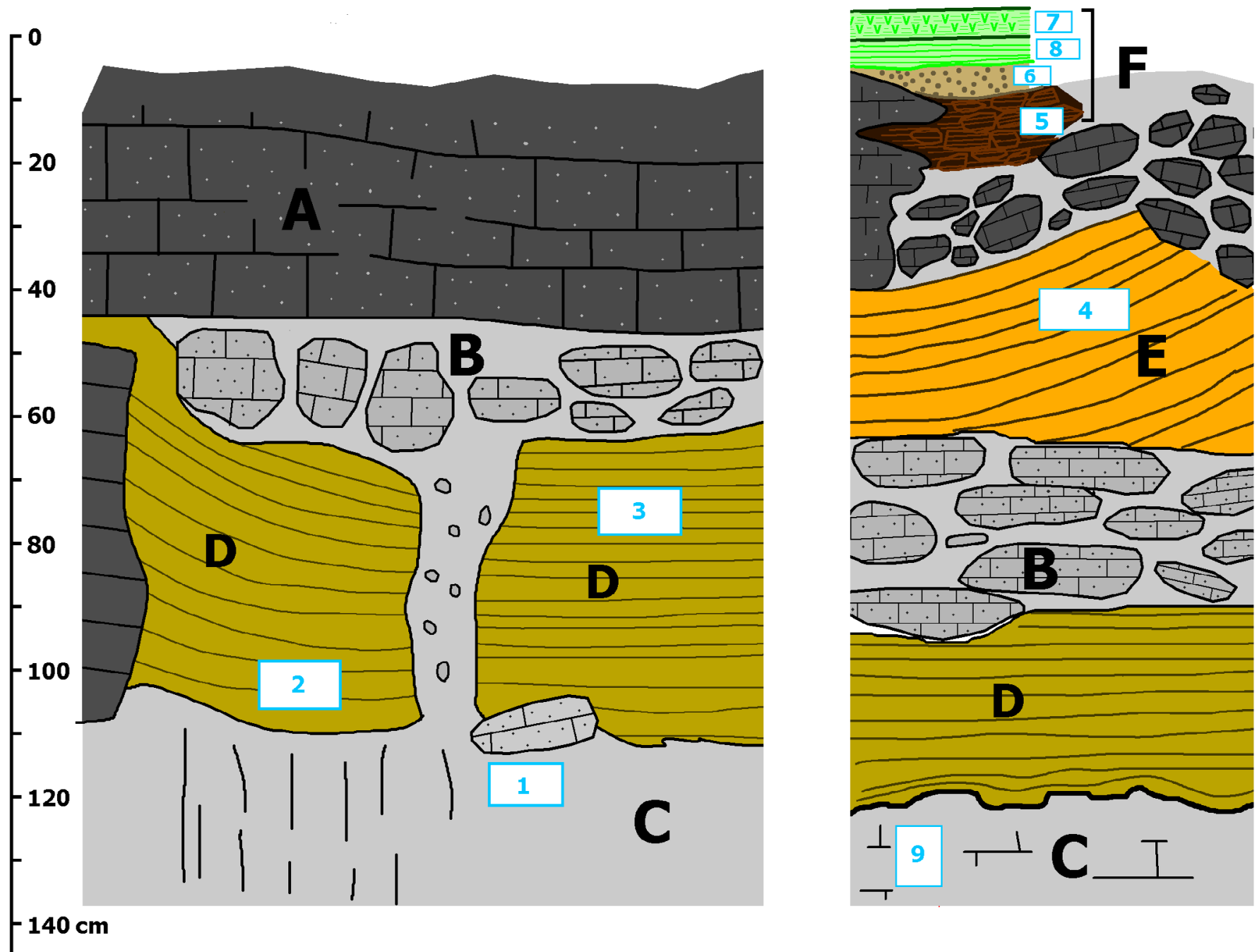

Fig. 8. Coupe lithostratigraphique du «coquelicot». Les numéros correspondent aux 9 échantillons prélevés. A. Roche mère un peu fantômisée. B. Blocs fantômisés dans une matrice d'altérite plus meuble. C. Altérite meuble. D. Formation argilo-limoneuse jaune stratifiée. E. Formation argileuse jaune sombre stratifiée. F. Ensemble supérieur comprenant: (5) une argile à blocaux, (6) un dépôt d'entroques libres, (7 et 8 ) une formation stalagmitique.

Fig. 8. Lithostratigraphic "coquelicot" section. The numbers correspond to the 9 removed samples. A. Bed-rock not very weathered. B. Weathered blocks in a weathered crumbly matrix. C. Crumbly alterite. D. Clayed-loamy yellow stratified formation. E. Darkly yellow clayed stratified formation. F. Upper unit composed by: (5) a clayed formation, (6) a free-crinoids body and (7 et 8) a speleothem.

d'une dynamique de sédimentation assez récente. Bien qu'ils aient $\mathrm{pu}$ théoriquement se développer longtemps après la sédimentation détritique sous-jacente, il est plus probable de conclure à une relative continuité entre le remplissage détritique et le spéléothème concerné. L'érosion du fantôme de roche se situerait alors dans le stade isotopique 5 .

\subsection{La grotte « des deux Albert»}

À une cinquantaine de mètres à l'est de la grotte « Nico », le front de la carrière a ouvert une petite cavité constituée principalement d'un couloir d'une vingtaine de mètres de longueur, présentant en son milieu un petit puits étroit. $\mathrm{Sa}$ principale caractéristique qui en fait une cavité holotype est qu'elle est entièrement creusée dans le fantôme de roche. Toutes les parois sont friables, poreuses. Au fond, impéné- trable, une coupe de sédiments de type argilo-limoneux jaune obstrue la suite (Fig. 16).

Notons enfin que la voûte présente des coulées stalagmitiques, prouvant la relative ancienneté de la cavité. La vidange ne résulte pas des travaux de la carrière, mais d'une érosion naturelle. L'examen des formes ne montre pas de trace d'érosion fluviatile. Cette dernière semble donc ici absente, si l'on excepte les sédiments sablo-argileux du fond. La vidange de l'altérite résulte donc d'un soutirage à partir de niveaux inférieurs actifs, comme cela se produit dans divers exemples hennuyers (Quinif et Maire, 2009).

\section{Genèse des grottes spéléologiques}

Le site de la Carrière du Coreux est exemplaire parce qu'il montre clairement le passage de fantômes de roche à la grotte 


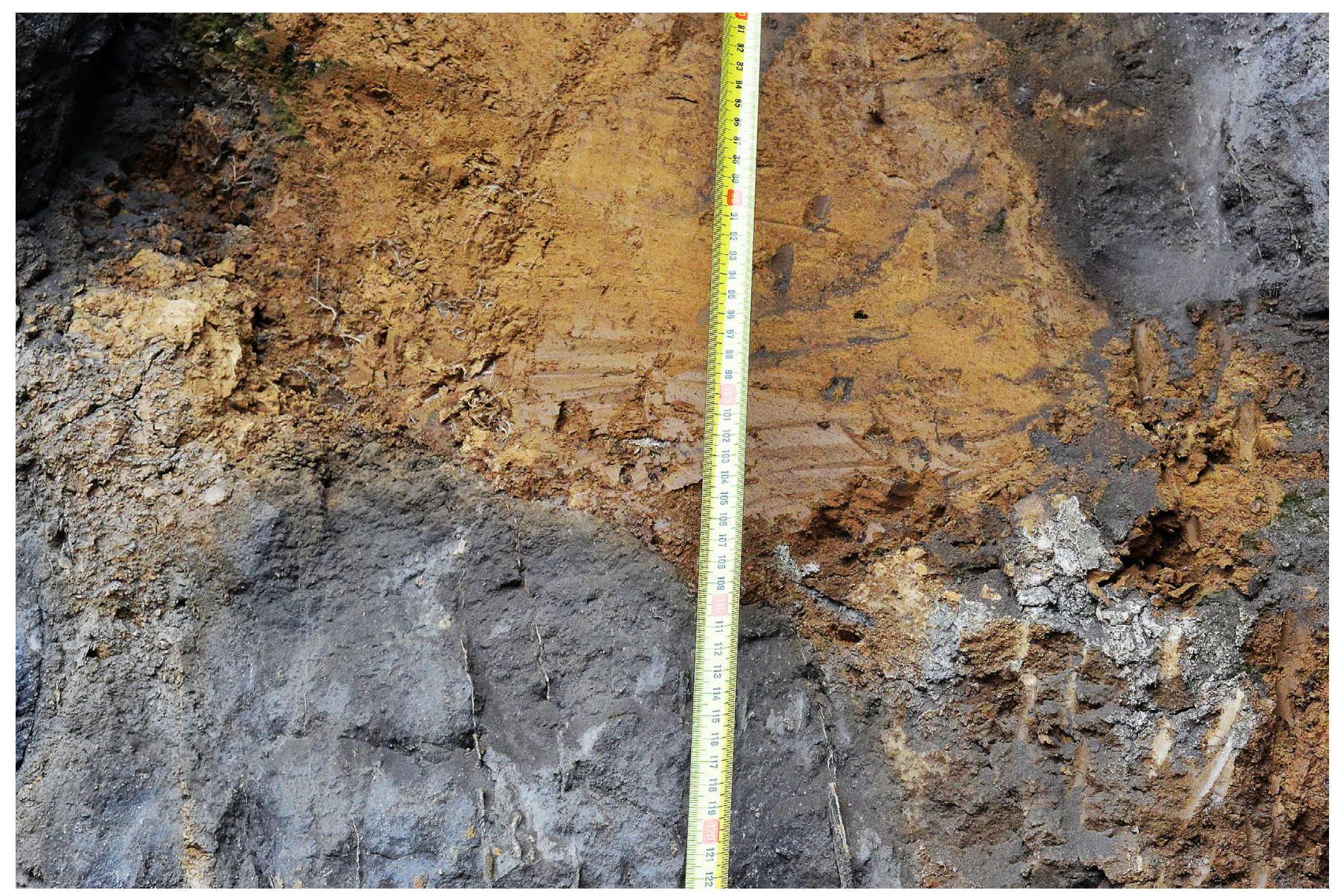

Fig. 9. Bas de la coupe de gauche. La formation gris-bleu basale est le fantôme de roche en place. On y distingue les fins filons de calcite subverticaux qui témoignent de l'altération in situ de la roche mère. Au-dessus, la formation est un limon argileux ravinant le fantôme de roche. Fig. 9. Bottom part of the left section. The grey bottom formation is the non-removed ghost-rock. One can see thin calcite vertical joints which are the witnesses of the in situ weathering of the bedrock. To the top, the clayed loamy formation is incising the ghost-rock.

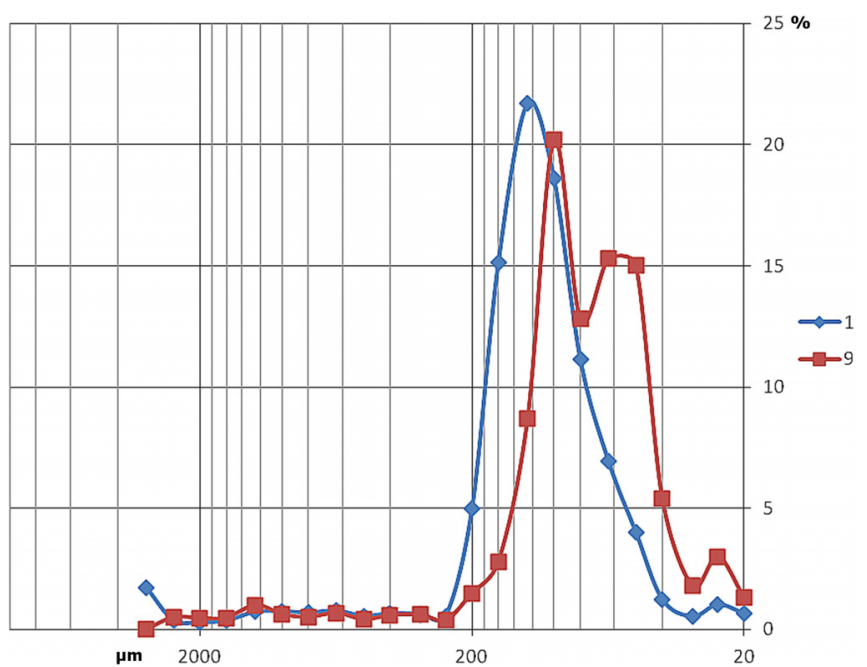

Fig. 10. Courbes granulométriques des deux échantillons d'altérite in situ. Les différences entre les deux tiennent à la texture de la roche mère.

Fig. 10. Granulometric curves of two samples of in situ alterite. The differences between the two samples are due to the bedrock texture. spéléologique au travers de coupes sédimentaires et de formes caractéristiques. La notion de fantôme de roche, introduite au début des années 1990, est fondée sur la notion de dissolution sélective de la roche mère, même en roches modérément solubles comme les calcaires. En conditions phréatiques avec un faible potentiel hydrodynamique, la circulation s'effectue en grandes boucles bathyphréatiques au travers de chemins à perméabilité initiale plus importante (joints en tectonique d'extension, strates poreuses, anciennes cavités), suffisante pour que la circulation s'établisse (Ford et Williams, 2007 ; Quinif, 1999). Dans ces conditions énergétiques, l'altération affecte une partie de la roche mère, laissant sur place une altérite résiduelle isovolume dans cette première phase évolutive. Cette première phase s'est vue fossilisée dans les calcaires dinantiens hennuyers et peut ainsi être observée dans les carrières (Quinif, 2010 ; Quinif et al., 1993, 2014 ; Vergari, 1996, 1998). La fantômisation affecte des formes souterraines initiées depuis la surface sous forme de couloirs verticaux ou totalement incluses dans la roche mère sous forme de volume remplis d'altérite résiduelle.

Le chemin conduisant des fantômes de roche aux grottes spéléologiques passe par une rupture d'équilibre de type biorhexistasique. L'apparition d'un potentiel hydrodynamique par soulèvement de la région et/ou abaissement de la surface 
Tableau 2. Composition minéralogique des échantillons (DRX sur poudres).

Table 2. Mineralogical composition of the samples (XRD on powder).

\begin{tabular}{lllllll}
\hline Échantillons & Dolomite (\%) & Quartz (\%) & Calcite (\%) & Muscovite (\%) & Smectite/chlorite & Kaolinite (\%) \\
\hline 1 (sur poudre) & 75 & 17,5 & 7,5 & 0 & 0 & 0 \\
9 (sur poudre) & 85 & 15 & & 0 & 0 & 5 \\
2 (sur dépôt) & 0 & 80 & 0 & 10 & 5 & 5 \\
3 (sur dépôt) & 0 & 80 & 0 & 0 & 0 & 0 \\
6 (sur poudre) & 75 & 20 & 0 & 0 & 5 \\
\hline
\end{tabular}

Tableau 3. Paramètres granulométriques de la formation argilo-limoneuse jaune.

Table 3. Granulometric parameters of the yellow clayed-loamy formation.

\begin{tabular}{lllllllll}
\hline Échantillon & $\begin{array}{l}\text { Refus à } \\
50 \mu \mathrm{m}(\%)\end{array}$ & $\begin{array}{l}\text { Fractile } \\
1(\mu \mathrm{m})\end{array}$ & $\begin{array}{l}\text { Médiane } \\
(\mu \mathrm{m})\end{array}$ & Qdphi & $\begin{array}{l}\text { Moyenne } \\
(\mu \mathrm{m})\end{array}$ & $\begin{array}{l}\text { Écart-type } \\
(\mu \mathrm{m})\end{array}$ & $\begin{array}{l}\text { Sig/moy } \\
(\%)\end{array}$ & $\begin{array}{l}\text { Skewness } \\
\text { Curtosis }\end{array}$ \\
\hline 2 & 3 & 142 & 11 & 0,98 & 16 & 25 & 156 & 7,9 \\
3 & 9 & 75 & 24 & 0,54 & 28 & 18 & 64 & 7,2 \\
\hline
\end{tabular}

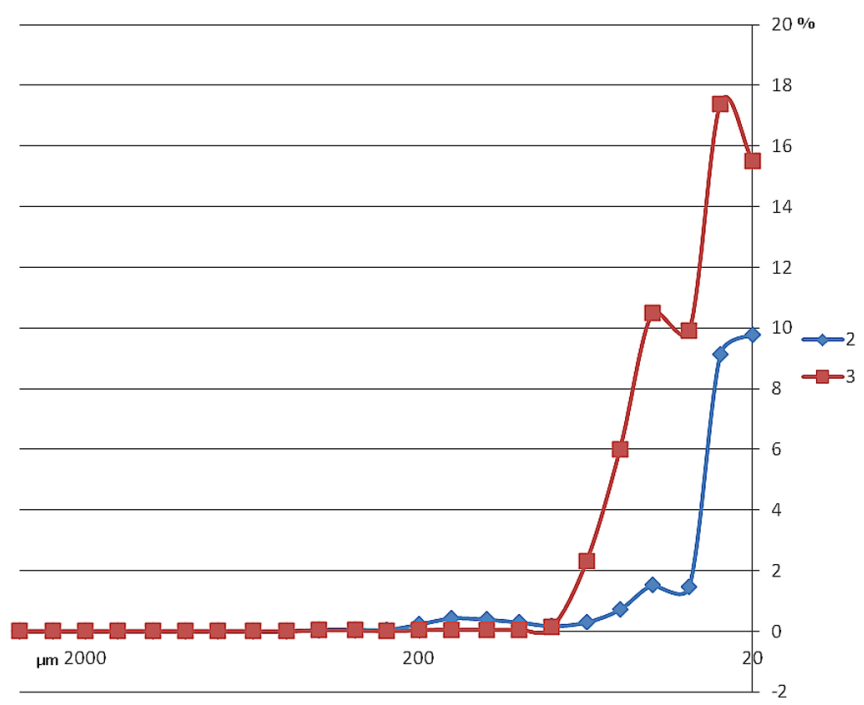

Fig. 11. Courbes granulométriques de la formation argilo-limoneuse jaune.

Fig. 11. Granulometric curves of the yellow clayed-loamy formation.

piézométrique permet l'apparition de circulations souterraines à plus forte énergie entre zone d'alimentation et zone d'exutoire située à plus basse altitude. Ces circulations au travers de l'altérite ont été prouvées dans les karsts du Hainaut (Quinif et Maire, 2009). Ces circulations peuvent éroder mécaniquement l'altérite résiduelle, ce qui dégage ainsi une cavité entre perte et résurgence. Cette jonction humainement pénétrable a été découverte en Entre-Deux-Mers (Dubois et al., 2011).

Les karsts belges ont connu ce schéma évolutif. Ces étapes ont pu être replacées dans l'évolution géologique du pays:

- après la tectogenèse varisque qui a essentiellement charrié et plissé le socle dévono-carbonifère en bordure nord de l'orogène, la pénéplanation de la chaîne de montagne aboutit à un relief peu prononcé durant l'ère mésozoïque. Essentiellement au début du Crétacé, un climat de type tropical altère vigoureusement les roches sous couvert végétal dense et favorise la mise en place de fantômes de roche en socle calcaire, ainsi que de puissantes altérites sur le socle dévonien quartzo-pélitique. En Hainaut, les grandes carrières des régions de Tournai et de Soignies permettent d'investiguer ces fantômes de roche transformés en paléokarsts par la transgression cénomanienne (Vergari et Quinif, 1997);

- dans les calcaires de Haute Belgique, cette fantômisation est fossilisée par les transgressions du Paléogène;

- lors de la surrection néogène, le réseau hydrographique s'enfonce de façon épigénétique dans le socle, créant ainsi le potentiel hydrodynamique permettant la vidange partielle des fantômes de roche. C'est à cette époque que se structure les réseaux de type pertes de plateaurésurgence de vallée analogue à celui de Sprimont. Cette évolution est synthétisée dans les figures $17 \mathrm{a}-\mathrm{c}$.

\section{Conclusion}

Le paradigme de la karstification par fantômisation ouvre de nouvelles perspectives sur la spéléogenèse, notamment dans le contexte de la Haute Belgique. La fantômisation consiste en une altération partielle de la roche mère en contexte biostasique avec conservation isovolumique de l'altérite résiduelle pendant une première phase. Celle-ci est remarquablement présente dans les carrières en Hainaut où elle a été fossilisée par les transgressions au Crétacé et au Paléocène (Quinif, 2010). La deuxième phase consiste en l'érosion mécanique de cette altérite lors d'une rhexistasie, causée par l'apparition d'un potentiel hydrodynamique suite à une surrection et un enfoncement des rivières. Les grottes spéléologiques apparaissent alors par vidange de l'altérite, 


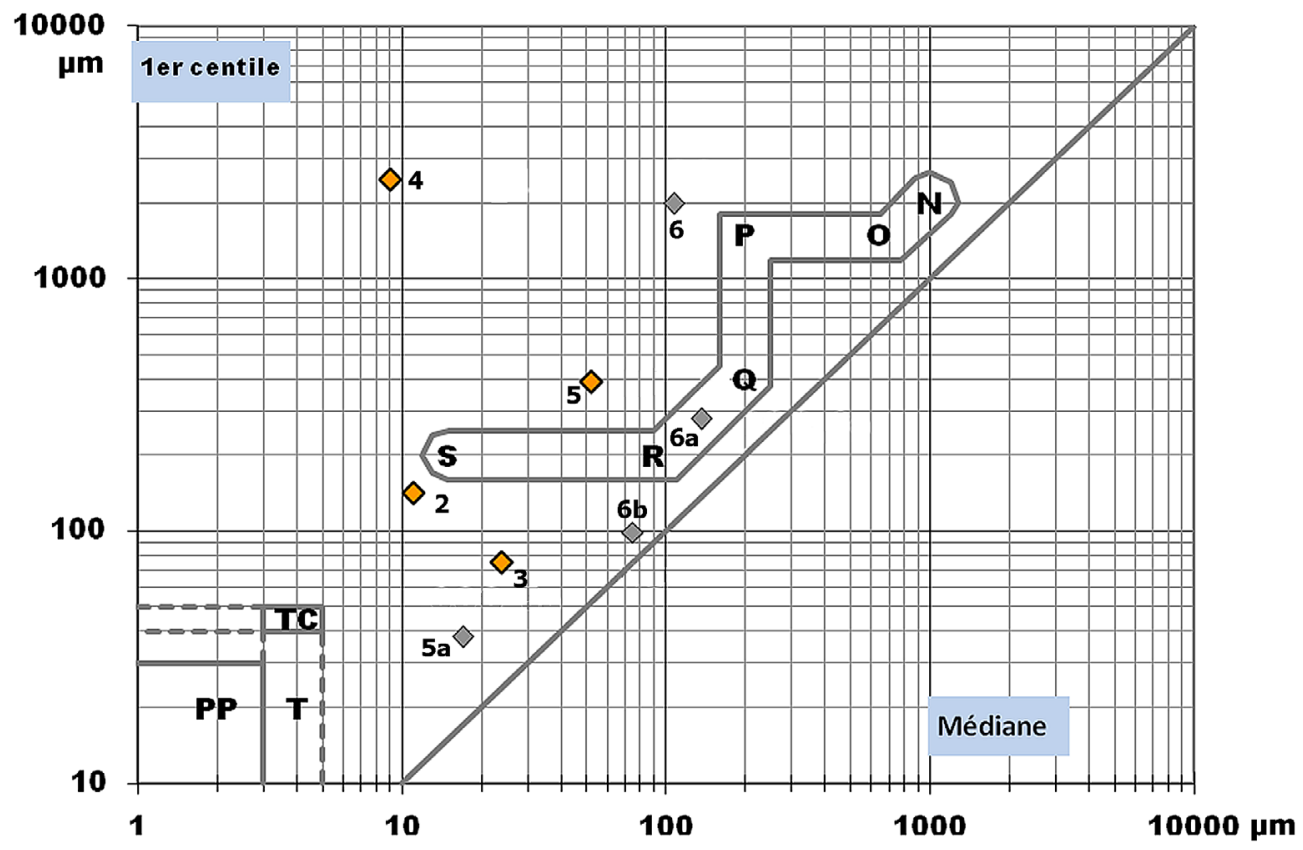

Fig. 12. Diagramme de Passega. SR : dépôt de suspension uniforme trahissant une vitesse de fond trop faible pour produire un classement quelconque (exemple : dépôts de lits majeurs). RQ : dépôt de suspension gradée apparaissant lorsque la charge dépasse la capacité de transport. QP : dépôt de sédiments transportés par roulement, avec une part de suspension (courant pas encore assez capacitif). PO : dépôt comprenant de plus en plus de grains roulés. ON : dépôt dont seuls subsistent les grains roulés, les suspensions étant totalement entraînées. Les surfaces PP, TC et $\mathrm{T}$ correspondent à des dépôts très fins de décantation totale.

Fig. 12. Passega's diagram. SR: uniform suspension deposit with a too weak bottom speed to make a grading, like major bed river deposits. RQ: graded suspension when the loading exceeds the transport capacity. QP: Rolling deposit, with a part of suspension. PO: Deposit with more and more rolling grains. ON: Rolling deposit without suspension. The areas PP, TC and T correspond to very thin deposits of total settling.

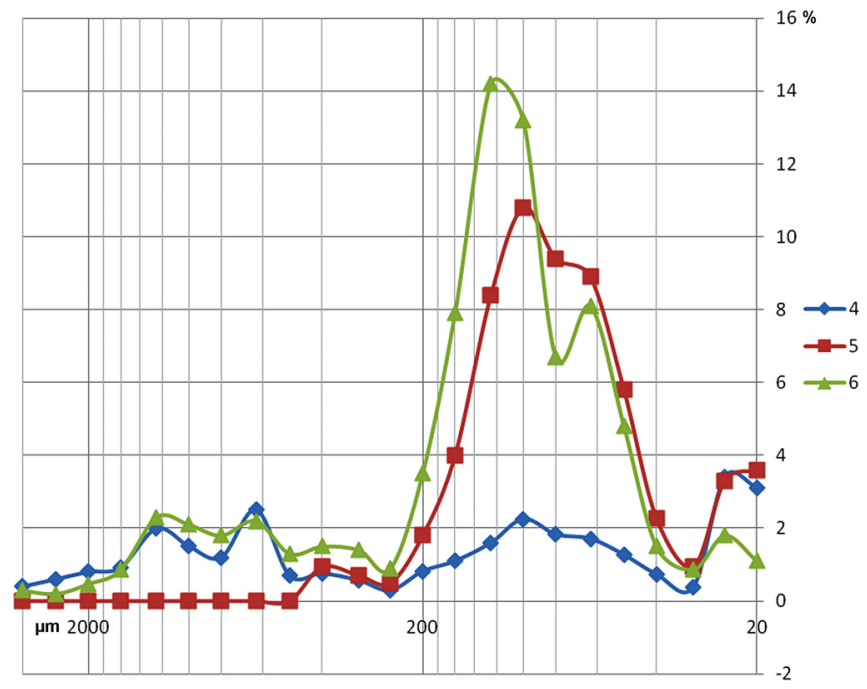

Fig. 13. Courbes granulométriques des échantillons détritiques supérieurs (coupe de droite). On remarque un certain parallélisme entre ces courbes.

Fig. 13. Granulometric curves of the upper detrital samples in the right section. On can see a parallelism between the curves. 


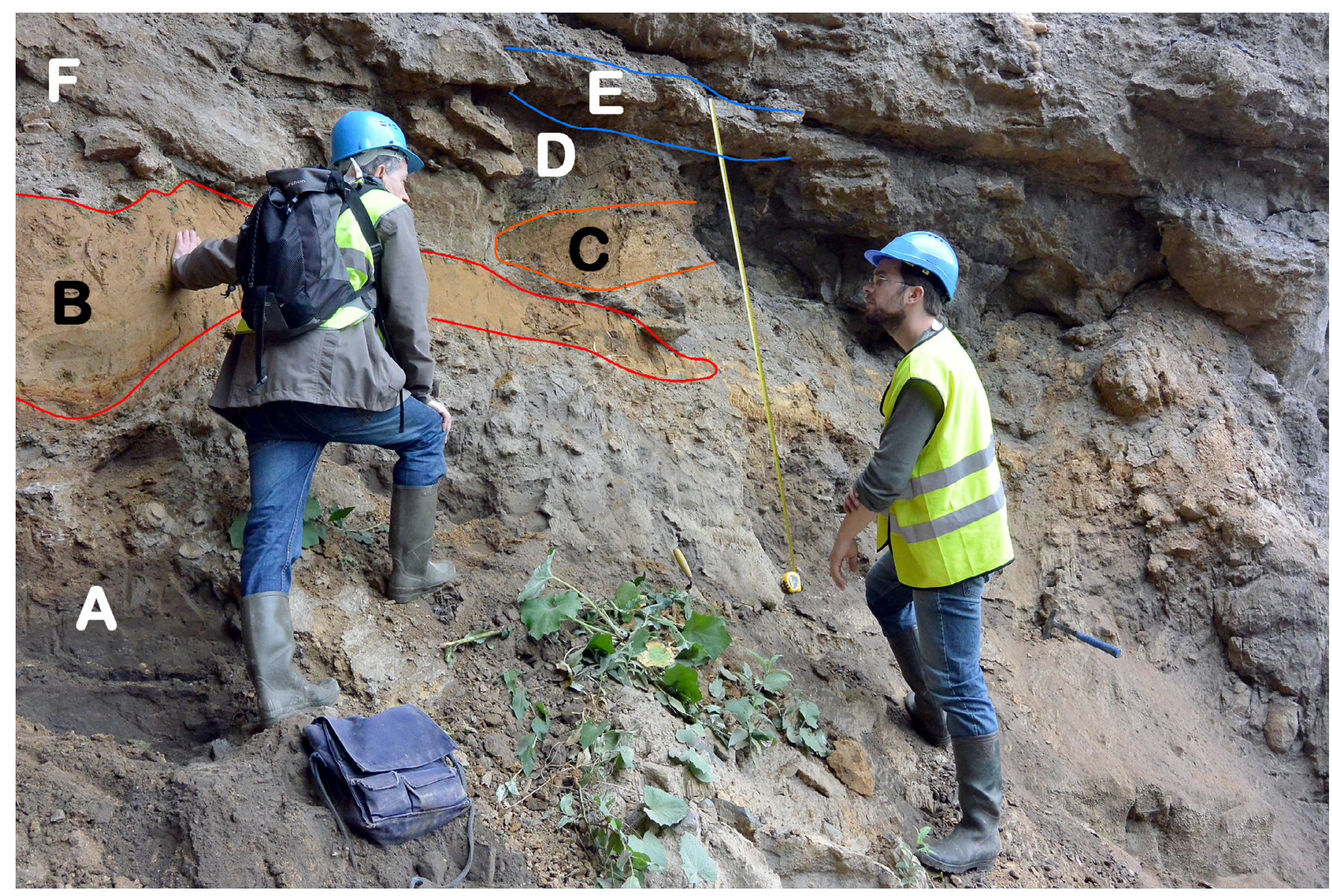

Fig. 14. Panorama de la coupe de droite. En A, sur toute la largeur de la photo, on trouve le fantôme de roche. B représente la formation argilolimoneuse. On voit qu'elle se réduit vers la droite tandis qu'à gauche, on la devine se continuer de façon plus importante. En C, la formation des limons ocre est coiffée par une bande de blocs fantômisés, puis des deux formations détritiques supérieures (D). E est le plancher stalagmitique qui coiffe tout l'ensemble. En F, une strate démantelée constitue le toit de la coupe de gauche.

Fig. 14. Panorama of the right section. The ghost-rock is in A, from the left to the right of the photo. B is the clayed-loamy formation which decreases to right. In $C$, the ocher loamy formation is capped by ghost-rock blocks and the two upper detrital formations (D). E is the top flowstone. The roof of the link section is constituted by a ghost-rock stratum.

Tableau 4. Paramètres granulométriques des formations de la coupe de droite.

Table 4. Granulometric parameters of the right section formations.

\begin{tabular}{|c|c|c|c|c|c|c|c|c|c|c|}
\hline Échantillon & $\begin{array}{l}\text { Mode } \\
(\mu \mathrm{m})\end{array}$ & $\begin{array}{l}\text { Refus à } \\
50 \mu \mathrm{m}(\%)\end{array}$ & $\begin{array}{l}\text { Fractile } \\
1(\mu \mathrm{m})\end{array}$ & $\begin{array}{l}\text { Médiane } \\
(\mu \mathrm{m})\end{array}$ & Qdphi & $\begin{array}{l}\text { Moyenne } \\
(\mu \mathrm{m})\end{array}$ & $\begin{array}{l}\text { Écart-type } \\
(\mu \mathrm{m})\end{array}$ & $\begin{array}{l}\text { Sig/ } \\
\operatorname{moy}(\%)\end{array}$ & Skewness & Curtosis \\
\hline 4 & & 42,41 & 2478 & 9 & 1,4 & 165 & 471 & 285 & 4 & 22 \\
\hline $5 a$ & $112(54 \%)$ & & 38 & 17 & 0,46 & 18 & 7 & & 0,6 & 2,6 \\
\hline 6 & & 74 & 1977 & 107 & 1 & 225 & 410 & 182 & 3,9 & 22 \\
\hline
\end{tabular}




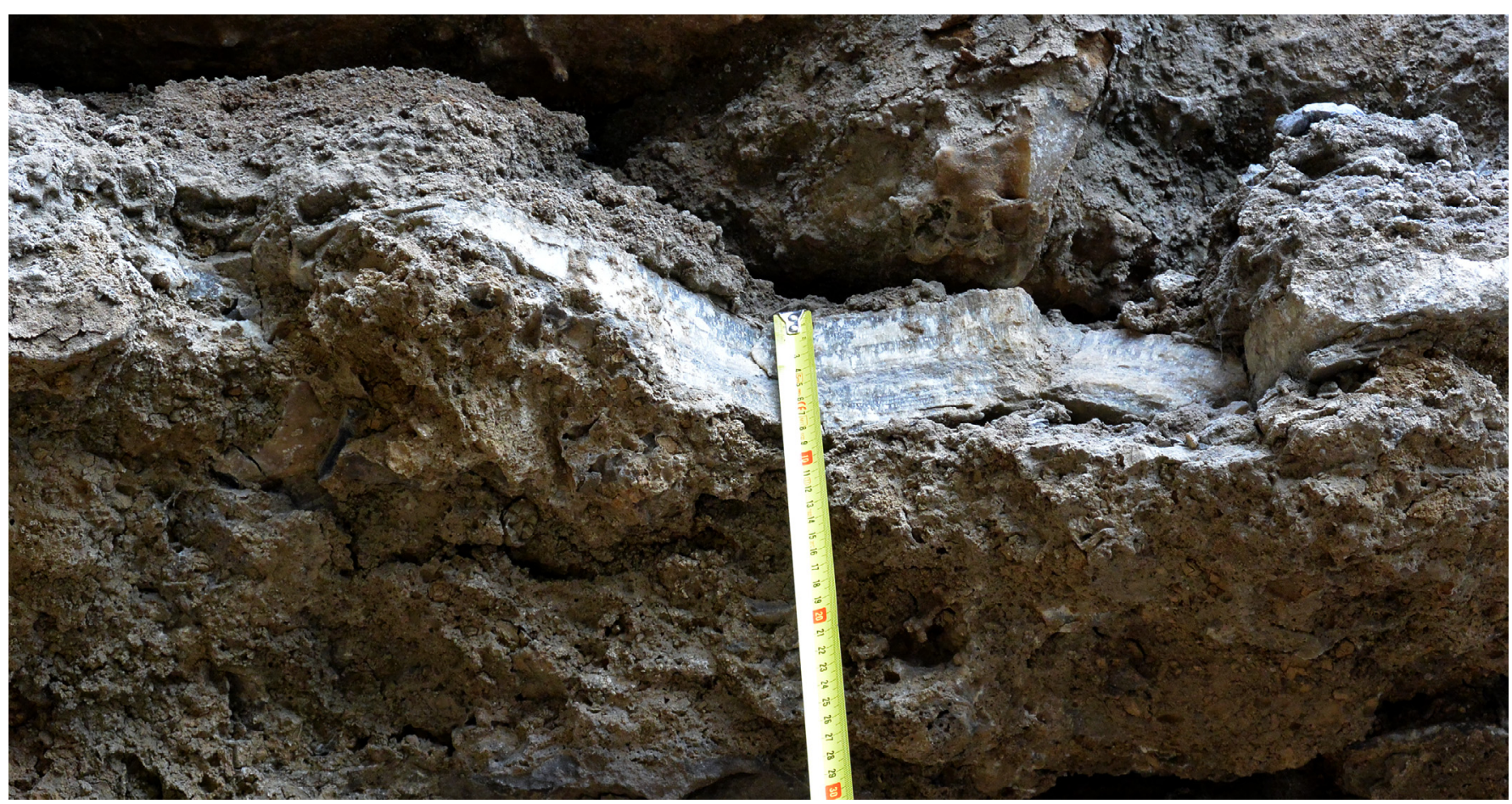

Fig. 15. Plancher stalagmitique terminal. Il s'appuie soit sur des sédiments, soit sur l'altérite résiduelle suivant l'endroit.

Fig. 15. Upper flowstone. It is laying either on sediments, either on the residual alterite.

Tableau 5. Datations du plancher sommital. 7 est le plancher supérieur, 8 le plancher inférieur. Les constantes de décroissance sont: $\lambda_{238}=1,55125 \times 10^{-10}$ (Jaffey et al., 1971), $\lambda_{234}=2,82206 \times 10^{-6}$ (Cheng et al., 2013), $\lambda_{230}=9,1705 \times 10^{-6}$ (Cheng et al., 2013). Les concentrations en ${ }^{232} \mathrm{Th}$ sont très faibles; néanmoins, une correction a été appliquée sur l'âge.

Table 5. Datings of the upper flowstone. 7 is the upper flowstone, 8 is the lower flowstone. The decay constants are $\lambda_{238}=1.55125 \times 10^{-10}$ (Jaffey et al., 1971), $\lambda_{234}=2.82206 \times 10^{-6}$ (Cheng et al., 2013), $\lambda_{230}=9.1705 \times 10^{-6}$ (Cheng et al., 2013). The concentrations in ${ }^{232}$ Th are very weak. Nevertheless, the ages are corrected.

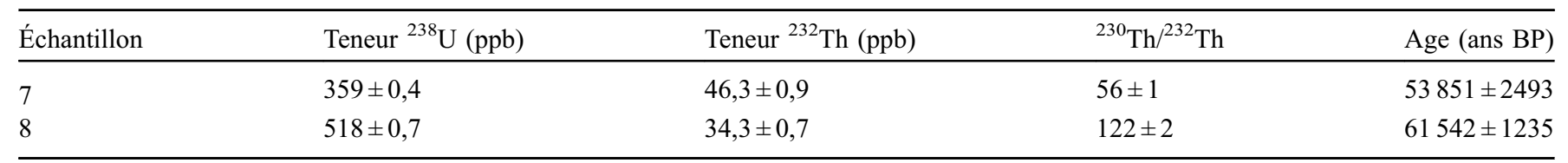

partielle ou en totale. Cette dynamique a pu être prouvée par la formation de la grotte «Quentin » à la Carrière de Nocarcentre (Écaussinnes, Hainaut, Belgique) suite à l'exhaure qui a créé un potentiel hydrodynamique artificiel (Quinif et Maire, 2009). Elle a aussi pu être observée dans le cadre d'un karst fossile à la Carrière du Clypot où un pseudoendokarst a été vidé partiellement de son altérite, remplacée par une série fluviatile durant le Dano-Montien (Quinif et al., 2006). Restait à prouver ce mécanisme dans le cadre d'un karst fonctionnel comme celui de la Haute Belgique. Le site de la carrière du Coreux exposé ici répond à cette question en prouvant la réalité de la spéléogenèse par érosion mécanique de l'altérite résiduelle d'un karst de type fantôme de roche. De plus, l'étude des dépôts prouve que ce mécanisme s'inscrit ici dans le Pléistocène supérieur pour l'amont du système.

Remerciements. Les auteurs tiennent à remercier Monsieur Victor Brancaleoni, administrateur-délégué des Carrières de Sprimont et de Chanxhe S.A., ainsi que le personnel de la carrière du Coreux pour leur autorisation et compréhension. Nous n'oublions pas non plus nos amis spéléologues Albert Briffoz et Albert Dubois dont les travaux ont contribué à la connaissance du sous-sol de cette région, ainsi que leur contribution à de longues discussions constructives. 

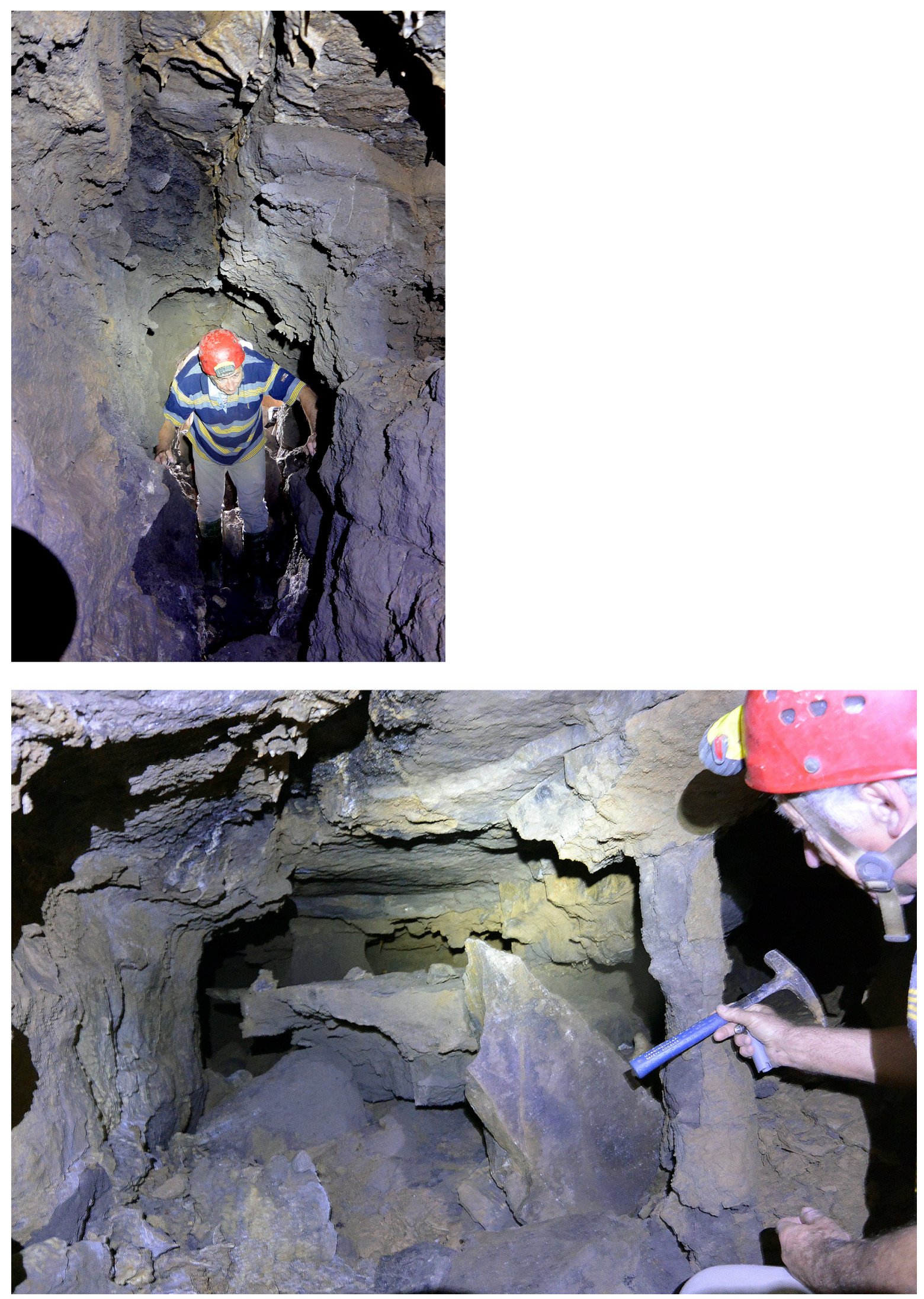

Fig. 16. La grotte «des deux Albert». Les formes déchiquetées résultent de l'érosion de l'altérite résiduelle.

Fig. 16. The cave of "deux Albert". The shredded shapes are due to the erosion of the residual alterite. 

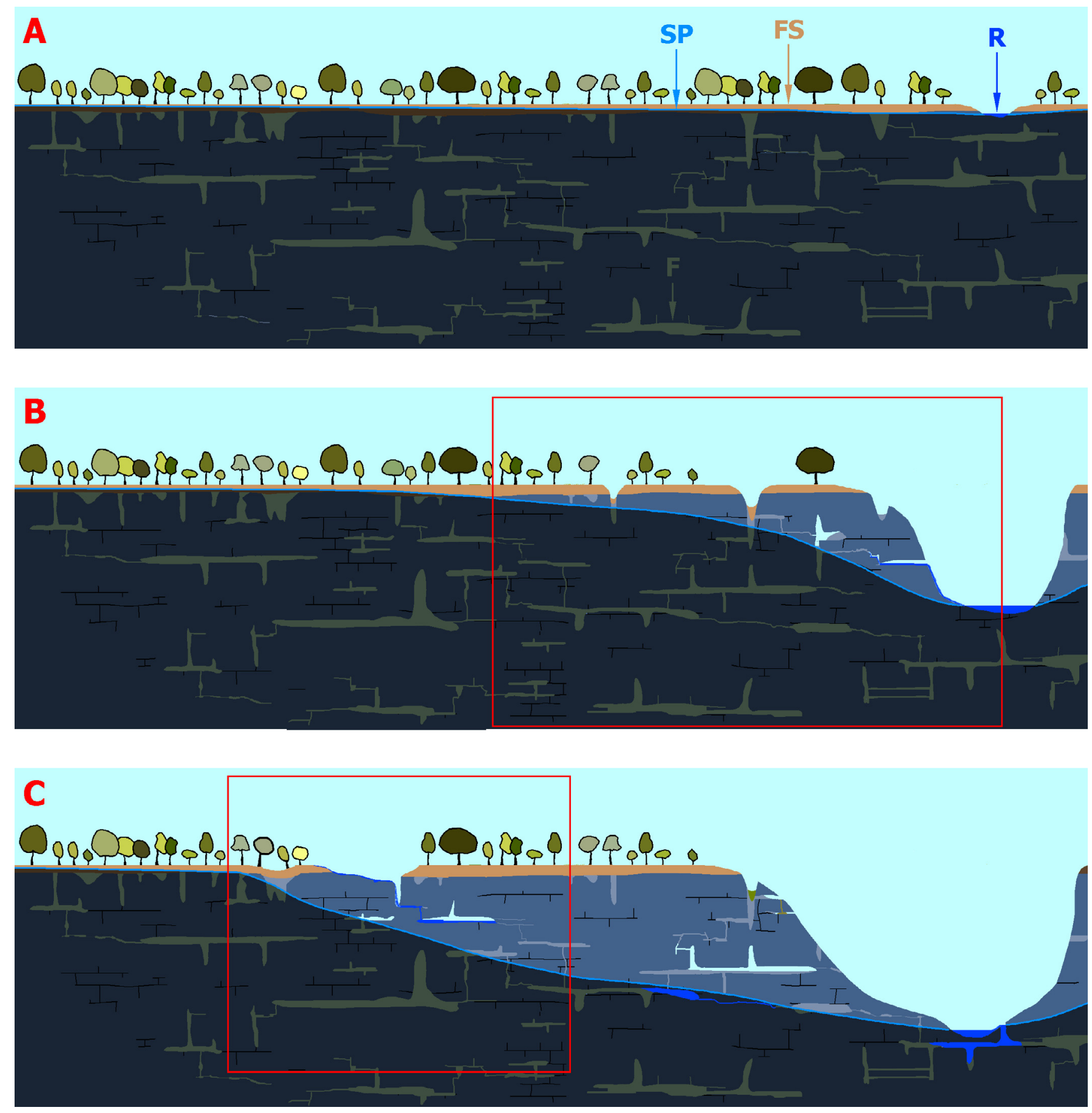

Fig. 17a. Schéma évolutif d'un massif karstifié par fantômisation. En A, un massif calcaire aplani supporte une formation superficielle (formation transgressive, zone profondément altérée, FS) qui est couverte d'une forêt. La rivière (R) qui impose le niveau de base et, donc, la surface piézométrique de l'aquifère (SP) est peu imprimée dans le paysage. Le potentiel hydrodynamique est faible ce qui entraîne des circulations souterraines très lentes. Suivant ce qui a été mis en évidence dans les paragraphes précédents, seule l'altération chimique agit, à l'exclusion de toute érosion mécanique (période biostasique). Le massif se fantômise ( $\mathrm{F}$, zones gris-vert). L'altération, suivant les lignes de courant, profite de toutes les fractures et des strates plus perméables pour transformer la roche mère en fantôme de roche. La distribution géométrique de ces fantômes n'est pas dictée par l'écoulement mais par l'allure des zones à perméabilité initiale plus forte. En B, le massif s'étant soulevé, la rivière s'est incisée et a abaissé la surface piézométrique dans sa zone d'influence. Le cadre rouge détaille les phénomènes proches du versant (Fig. 17b : vidange de fantômes de roche, résurgence, formation de puits en surface. L'allure de la surface piézométrique est ici purement indicative et dépend de la distribution de la perméabilité au sein du massif fantômisé. En C, l'incision fluviatile s'est accentuée. Le recoupement d'un fantôme de roche profond a conduit à la formation d'une source vauclusienne. Un certain volume de l'altérite résiduelle a été érodé (exemple des sources de la Touvre, en Charente). Le cadre rouge détaille des phénomènes en amont du système (Fig. 17c) : tassement et érosion de l'altérite sur le parcours d'une circulation souterraine, perte, ouverture de grottes. 


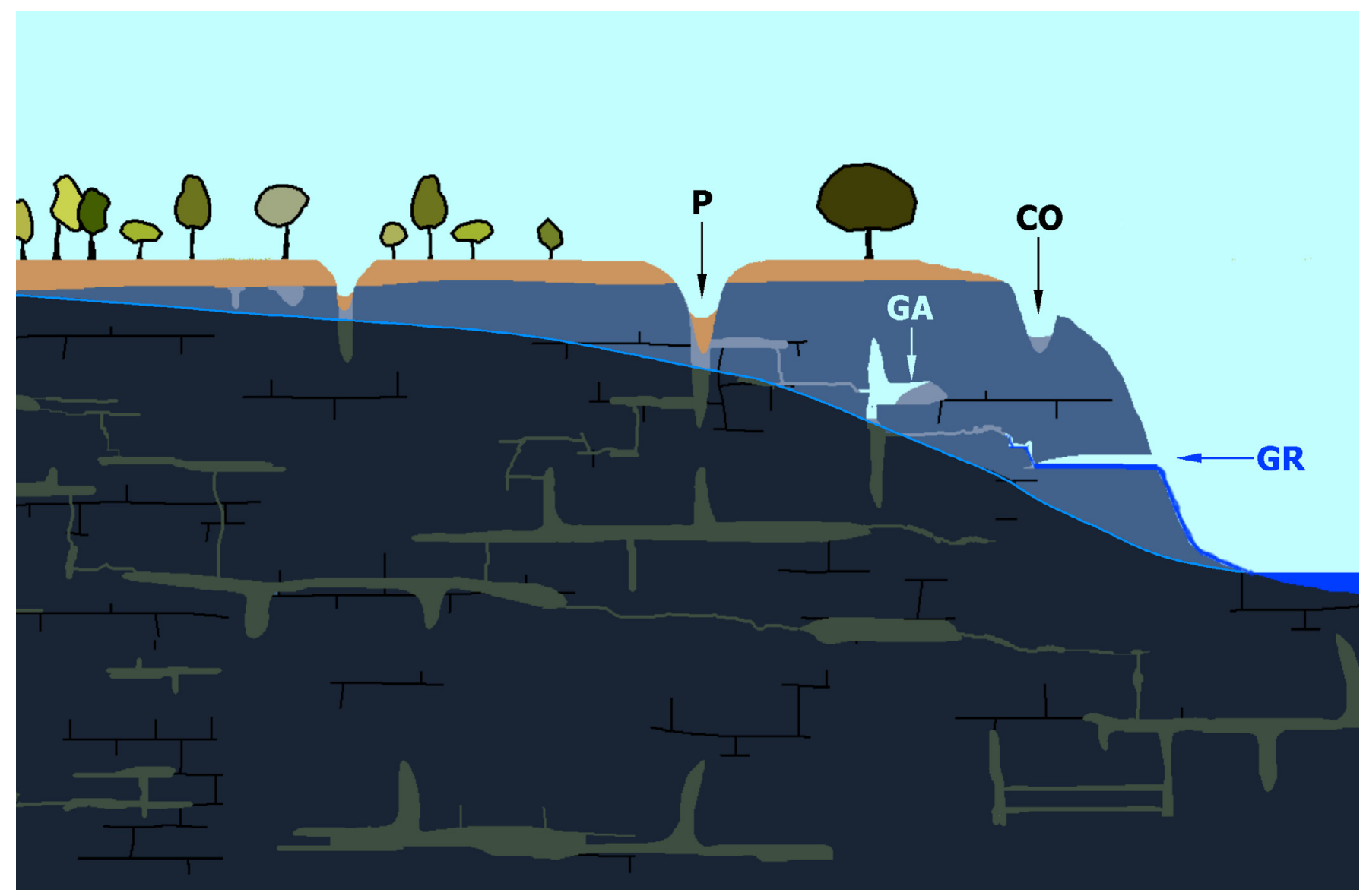

Fig. 17b. Agrandissement du premier cadre rouge. La surface piézométrique, en s'abaissant, a placé un coin du massif en zone vadose. Une circulation au travers de l'altérite s'est établie entre la surface et le versant. Cette circulation a vidé partiellement une série de fantômes de roche ce qui a ouvert une grotte résurgence (GR; exemple des résurgences de Val Imagna en Lombardie, Italie). Le tassement de l'altérite résiduelle a provoqué un effondrement de surface avec apparition d'un puits (P), ainsi que l'ouverture de vides internes sous forme de grotte aveugle (GA). Un ancien couloir $(\mathrm{CO})$ a été partiellement vidé de son altérite et peut jouer un rôle de piège à sédiments. Remarquons que l'altitude de la grotte résurgence n'est pas dictée par un niveau altitudinal de la rivière aérienne mais par la position préalable du fantôme de roche.

Fig. 17b. Enlargement of the first red box. The going down of the water table has provoked a part of the massif in the vadose zone. A flow through the alterite has been created between the surface and the sloop. This flow has opened a number of ghostrocks with the creation of a resurgence cave (GR). The collapse of the alterite is at the origin of a collapse pit at the surface ( $P$ ) and the opening of blind empty spaces (blind cave, GA). A vertical channel (CO) has been emptied and plays the role of sediments trap. The altitude of the resurgence is not dependant on an altitudinal level of the river but on the previous position of the ghostrock.

Fig. 17 a (Continued). Evolution of a karstified massif by the ghostrock process. In A, a limestone massif is covered by an overburden formation (transgressive formation, deeply weathered zone, FS) under a tropical forest. The river R is very weakly hollowed. The water table is thus very superficial. The hydrodynamical potential is weak; the underground flows are very slow. It is a biostasy phase with an intense chemical weathering without the mechanical erosion. The ghostrock karstification happened (F, grey-green areas). The water goes through the opened joints or strata: the initial permeability ways to transform the bedrock in ghostrocks. The geometry of the weathered areas is not dependent from the current lines but by the areas with greater initial permeability. In $B$, the massif is in surrection and the rivers are sinking. The water table is going down in the influence areas of the rivers. Red box details the phenomena which are in neighbor of the slope (Fig. 17b): emptying of the ghostrock by erosion of the residual alterite, resurgence, pit genesis at the surface. The appearance of the water table is purely indicative and depends on the geometry of the permeability into the rock massif. In $C$, the river incision grows. The intersection of a deep ghostrock conducts to the formation of a Vauclusian spring. More and more volumes of the residual alterite are eroded. The red box details the upper system phenomena (Fig. 17c): subsidence, collapse, erosion of the residual alterite along the course of an underground river, swallow holes, opening of caves. 


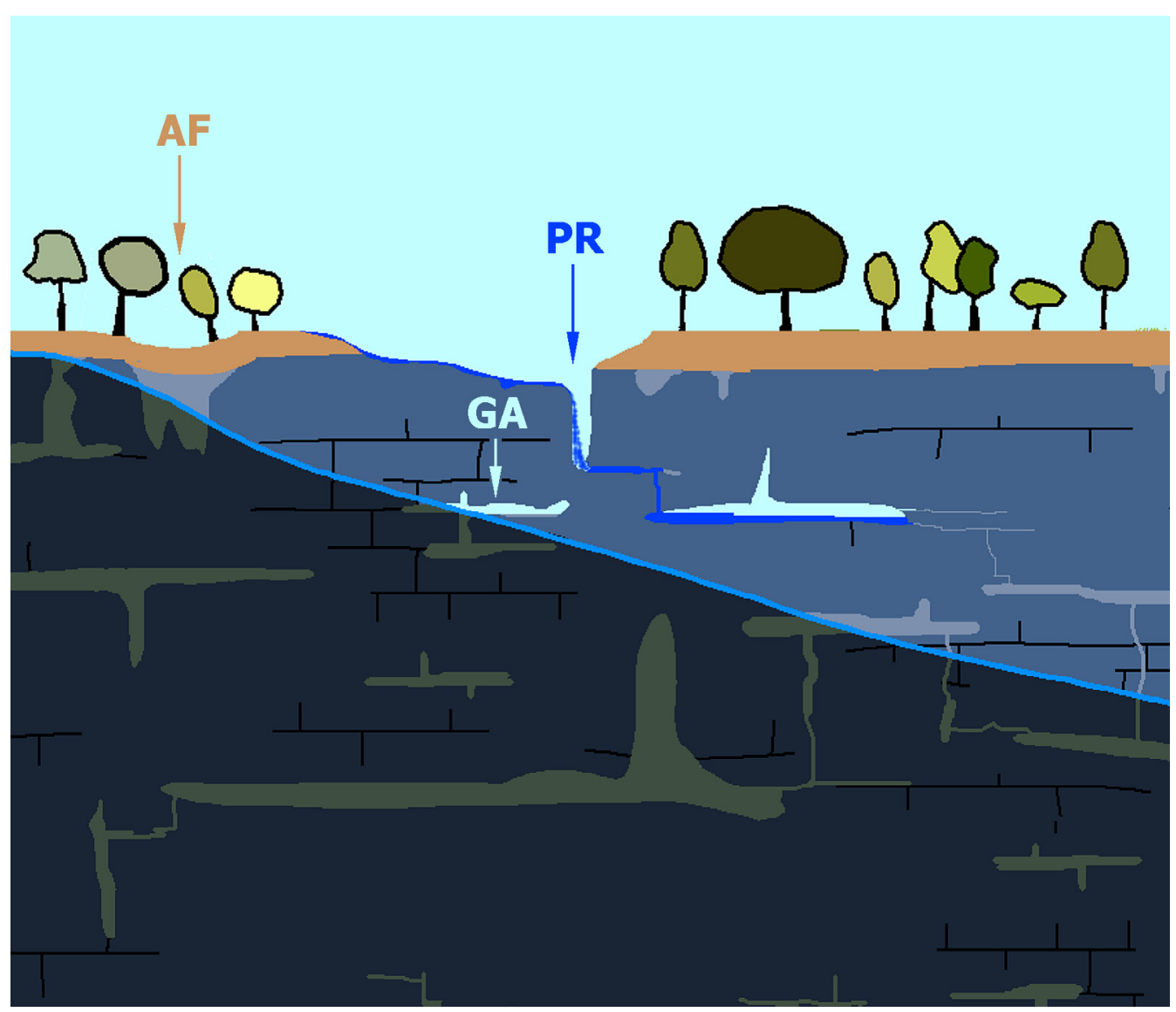

Fig. 17c. Agrandissement du second cadre rouge. Dans l'amont du système, une perte s'est formée (PR) par capture d'un ruisseau aérien dans un fantôme de roche vidé. L'érosion élimine de plus en plus d'altérite. La circulation s'est amorcée, qui évoluera vers une connexion perterésurgence, laquelle évoluera ensuite sur un mode fluviatile. Un affaissement s'amorce avec basculement des arbres et soutirage de la formation de couverture (AF).

Fig. 17c. Enlargement of the second red box. In the upstream of the system, a swallow hole has captured a surface river (PR) in an emptied ghostrock. The erosion brings more and more residual alterite. The underground flow has been initiated to a connection swallow hole-resurgence. After the erosion of the alterite, we obtain a speleological connection by an underground river. A collapse begins with a destabilization of trees and decanting of the overburden formation (AF).

\section{Références}

Bellière J, Marion J-M. 2009. Carte géologique de Wallonie, TavierEsneux (49/1-2), version provisoire. Namur: Ministère de la Région Wallonne.

Briffoz A. 1983. Sprimont: le sous-sol régional sous la loupe: Commission des publications de la Fédération Spéléologique Francophone de Belgique A.S.B.L. Cahiers du Spéléo Flash 3: 176.

Cheng H, Edwards RL, Shen C-C., Woodhead J, Hellstrom J, Wang YJ, Kong XG, et al. 2013. Improvements in ${ }^{230}$ Th dating, ${ }^{230} \mathrm{Th}$ and ${ }^{234} \mathrm{U}$ half-life values, and U-Th isotopic measurements by multicollector inductively coupled plasma mass spectrometry. Earth and Planet Sci Lett 371-372: 82-91.

De Broyer C, Thys G, Fairon J, Michel G, Vrolix M. 1996. Atlas du Karst Wallon-Province de Liège-Région Wallonne et Commission Wallonne d'Etude et de Protection des sites souterrains (C.W. E.P.S.S.), 3 tomes, 896 p.

Demoulin A. (Ed.). 1995. L'Ardenne. Essai de géographie physique. Département de Géographie physique et Quaternaire, Université de Liège, $238 \mathrm{p}$.
Dubois C, Lans B, Kaufmann O, Maire R, Quinif Y. 2011. Karstification de type fantômes de roche en Entre-deux-Mers (Gironde, France): implications en karstogenèse et morphologie karstique. Karstologia 57: 19-27.

Dubois C, Quinif Y, Baele J-M., Barriquand L, Bini A, Bruxelles L, et al. 2014a. The process of ghost-rock karstification and its role in the formation of cave systems. Earth Science Reviews 131: 116148.

Dubois C, Quinif Y, Baele J-M., Dagrain F, Deceuster J, Kaufmann O. 2014b. The evolution of the mineralogical and petrophysical properties of a weathered limestone in southern Belgium. Geologica Belgica 17(1): 1-8.

Dubois J. 1982. Cavités karstiques de la Province de Liège, inventaire des phénomènes karstiques pénétrables tels que: abîme, abri, caverne, chantoir, faille, grotte, puits et trou (première partie). Service géologique de Belgique, Professional Paper 1982/1, 187, $217 \mathrm{p}$.

Dupont N, Dubois C, Kaufmann O. 2012. Fracturation et fantômes de roche dans le système karstique de Chanxhe-Sprimont. In: Journées de la Spéléologie Scientifique, Centre belge d'Étude $d u$ Karst et Commission scientifique de l'Union belge de 
Spéléologie. Han-sur-Lesse, Belgique, 8/12/2012-9/12/2012, oral communication.

Dupont N, Quinif Y, Dubois C, Kaufmann O. 2016. Speleogenesis by alterite erosion within ghost-rock features in the Ardenne Allochton (Sprimont syncline, East Belgium). In: 5th International Geologica Belgica Meeting. Mons, Belgique, 26-29/01/2016, poster communication.

Ek C. 1957. Les terrasses de l'Ourthe et de l'Amblève inférieures. Annales de la Société géologique de Belgique 80: 334-353. Disponible sur http://hdl.handle.net/2268/143617.

Ek C. 1976. Les phénomènes karstiques. In: Géomorphologie de la Belgique. Hommage au Professeur Paul Macar. Laboratoire de Géologie et de Géographie physique de l'Université de Liège, pp. 137-157.

Ek C, Labarde P, Peeters A, Michel R. 2014a. Un synclinal dans le Calcaire Carbonifère. In: Gilles-Sépulchre Y, ed. Sprimont gravé dans la pierre. Sprimont: Brancaleoni, pp. 141-144.

Ek C, Willems L, Briffoz A, Boulanger J-M. 2014b. Nouvelles découvertes : le Noû Bleû. In: Gilles-Sépulchre Y, ed. Sprimont gravé dans la pierre. Sprimont : Brancaleoni, pp. 160-163.

Ford, DC, Williams P. 2007. Karst Hydrogeology and Geomorphology. Chichester: John Wiley \& Sons, 562 p.

Fourmarier P. 1954. Carte géologique de la Belgique, Louveigné-Spa $\left(n^{\circ} 148\right)$. Bruxelles: Service géologique de Belgique.

Havron C, Baele J-M., Quinif Y. 2007. Pétrographie d'une altérite résiduelle de type "fantôme de roche ". Karstologia 49: 25-32.

Jaffey AH, Flynn KF, Glendenin LE, Bentley WC, Essling AM. 1971. Precision measurements of half-lives and specific activities of 235U and 238U. Physical Reviews C 4: 1889-1906.

Meus P, Moureaux P, Gaillier S, Flament J, Delloye F, Nix P. 2014. In situ monitoring of karst springs in Wallonia (Southern Belgium). Environ Earth Sci 71: 533-541. DOI: 10.1007/s12665-013-2760-x.

Mottequin B, Marion JM. 2012. Lithostratigraphie du Tournaisien et du Viséen (Carbonifère) dans le synclinal de Chanxhe-Sprimont (province de Liège, Belgique). Journée de Spéléologie Scientifique. Centre belge de l'étude du Karst et Commission Scientifique de 1'Union belge de spéléologie. Disponible sur http://hdl.handle.net/ 2268/135801.

Mottequin B, Marion JM. 2014. Contribution scientifique et administrative : Cadre géologique. In: Gilles-Sépulchre Y, ed. Sprimont, gravé dans la pierre. Sprimont: Brancaleoni, pp. 181188. Disponible sur http://hdl.handle.net/2268/111946.

Nys L. 1929. Observations sur l'hydrologie des calcaires dans la région de Sprimont-Chanxhe. Annales de la Société Géologique de Belgique 52: 219-220.

Passega R. 1957. Texture as characteristic of clastic deposition. Bull Amer Ass Petrol Geol 41(9): 1952-1984.

Passega R. 1964. Grain size representation by CM patterns as a geological tool. Journ Sed Petr 34(4): 830-847.

Passega R. 1977. Significance of CM diagrams of sediments deposited by suspension. Sedimentology 24: 723-733.
Passega R, Byramjee R. 1969. Grain-size image of clastic deposits. Sedimentology 13: 233-252.

Peeters A, Ek C, Labarde P, Michel R. 2012. La dynamique du karst dans le synclinal de Sprimont. Excursion des Journées de la Spéléologie scientifique. Centre belge d'Étude du Karst et Commission scientifique de l'Union belge de Spéléologie, 1-6. Disponible sur http://hdl.handle.net/2268/184016.

Poty E, Hance, L, Lees A, Hennebert M. 2002. Dinantian lithostratigraphic units (Belgium). Geologica Belgica 4: 69-94.

Price DG. 2009. Engineering Geology: Principles and Practice. Berlin: Springer, $472 \mathrm{p}$.

Quinif Y. 1999. Fantômisation, cryptoaltération et altération sur roche nue, le triptyque de la karstification. Études de géographie physique, Travaux 1999-Supplement 18, University of Provence, pp. 159-164.

Quinif Y. 2010. Fantômes de roche et fantômisation-Essai sur un nouveau paradigme en karstogenèse. Karstologia Mémoires 18: 196.

Quinif Y, Maire R. 2009. La Grotte Quentin (Hainaut, Belgique) : un modèle d'évolution des fantômes de roche. Karstologia Mémoires 17: 214-218.

Quinif Y, Vergari A, Doremus P, Hennebert M, Charlet J-M. 1993. -Phénomènes karstiques affectant le calcaire du Hainaut. Bull Soc Belg Géol 102: 379-394.

Quinif Y, Meon H, Yans J. 2006. Nature and dating of karstic filling in the Hainaut Province (Belgium). Karstic, geodynamics and paleogeographic implications. Geodinamica Acta 19(2): 73-85.

Quinif Y, Baele J-M, Dubois C, Havron C, Kaufmann O, Vergari A. 2014. Fantômisation: un nouveau paradigme entre la théorie des deux phases de Davis et la théorie de la biorhexistasie d'Erhart. Geologica Belgica 17(1): 66-74.

Ruthy I. 2004. Carte hydrogéologique de Wallonie, planchette TavierEsneux (49/1-2), notice explicative, version provisoire. Université de Liège, Département GéomaC, 43 p. + annexes.

SPW-DGARNE. 2005. Atlas du Karst Wallon. Données numériques accessibles sur le lien suivant: http://carto1.wallonie.be/CIGALE/ viewer.htm?APPNAME=OGEAD.

Van den Broeck E, Martel EA, Rahir E. 1910. Les cavernes et les rivières souterraines de la Belgique ( 2 tomes) : 1592 p. + annexes.

Vergari A. 1996. Contraintes paléokarstiques dans l'exploitation du calcaire carbonifère sur le bord nord du synclinorium de Namur en Hainaut occidental. Ph.D. Thesis, Faculté Polytechnique de Mons.

Vergari A. 1998. Nouveau regard sur la spéléogenèse: le pseudoendokarst du Tournaisis (Hainaut, Belgique). Karstologia 31: $12-18$.

Vergari, A. 2014. La grotte du Noû Bleû, un exemple unique de la naissance d'une grotte par réactivation de la fantômisation d'un massif calcaire. In : Gilles-Sépulchre Y, ed. Sprimont gravé dans la pierre. Sprimont: Brancaleoni, pp. 163-164.

Vergari A, Quinif Y. 1997. Les paléokarsts du Hainaut. Geodinamica Acta 10(4): 175-187.

Citation de l'article : Dupont N, Quinif Y, Dubois C, Cheng H, Kaufmann O. 2018. Le système karstique de Sprimont (Belgique). Holotype d'une spéléogenèse par fantômisation, BSGF - Earth Sciences Bulletin 189: 1. 